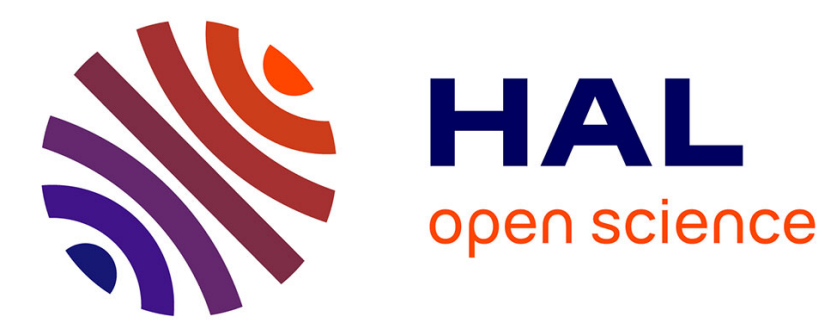

\title{
A posteriori stopping criteria for optimized Schwarz domain decomposition algorithms in mixed formulations
}

Sarah Ali Hassan, Caroline Japhet, Michel Kern, Martin Vohralík

\section{To cite this version:}

Sarah Ali Hassan, Caroline Japhet, Michel Kern, Martin Vohralík. A posteriori stopping criteria for optimized Schwarz domain decomposition algorithms in mixed formulations. Computational Methods in Applied Mathematics, 2018, 18 (3), pp.495-519. 10.1515/cmam-2018-0010 . hal-01529532v3

\section{HAL Id: hal-01529532 \\ https://hal.inria.fr/hal-01529532v3}

Submitted on 12 Jul 2018

HAL is a multi-disciplinary open access archive for the deposit and dissemination of scientific research documents, whether they are published or not. The documents may come from teaching and research institutions in France or abroad, or from public or private research centers.
L'archive ouverte pluridisciplinaire HAL, est destinée au dépôt et à la diffusion de documents scientifiques de niveau recherche, publiés ou non, émanant des établissements d'enseignement et de recherche français ou étrangers, des laboratoires publics ou privés. 


\title{
A posteriori stopping criteria for optimized Schwarz domain decomposition algorithms in mixed formulations*
}

\author{
Sarah Ali Hassan, Caroline Japhet ${ }^{\dagger}$ Michel Kern $\ddagger$ and Martin Vohralík ${ }^{\ddagger}$
}

June 28, 2018

\begin{abstract}
This paper develops a posteriori estimates for domain decomposition methods with optimized Robin transmission conditions on the interface between subdomains. We choose to demonstrate the methodology for mixed formulations, with a lowest-order Raviart-Thomas-Nédélec discretization, often used for heterogeneous and anisotropic porous media diffusion problems. Our estimators allow to distinguish the spatial discretization and the domain decomposition error components. We propose an adaptive domain decomposition algorithm wherein the iterations are stopped when the domain decomposition error does not affect significantly the overall error. Two main goals are thus achieved. First, a guaranteed bound on the overall error is obtained at each step of the domain decomposition algorithm. Second, important savings in terms of the number of domain decomposition iterations can be realized. Numerical experiments illustrate the efficiency of our estimates and the performance of the adaptive stopping criteria.
\end{abstract}

Key words: Heterogeneous diffusion, mixed finite element method, domain decomposition method, optimized Schwarz method, Robin transmission conditions, a posteriori error estimate, stopping criteria

\section{Introduction}

We consider in this paper the following model diffusion problem: find the fluid pressure head $p$ and the Darcy velocity $\mathbf{u}$ such that

$$
\begin{aligned}
& \mathbf{u}=-\boldsymbol{S} \nabla p \text { in } \Omega, \\
& \nabla \cdot \mathbf{u}=f \quad \text { in } \quad \Omega \text {, } \\
& p=g_{\mathrm{D}} \quad \text { on } \quad \Gamma^{\mathrm{D}} \text {, } \\
& -\mathbf{u} \cdot \mathbf{n}=g_{\mathrm{N}} \quad \text { on } \quad \Gamma^{\mathrm{N}},
\end{aligned}
$$

where $\Omega \subset \mathbb{R}^{d}, d=2,3$, is a polygonal (polyhedral if $d=3$ ) domain (open, bounded, and connected set) with Lipschitz-continuous boundary $\partial \Omega=\Gamma^{\mathrm{D}} \cup \Gamma^{\mathrm{N}}$. Here $\Gamma^{\mathrm{N}}$ is the boundary with a Neumann condition $g_{\mathrm{N}} \in L^{2}\left(\Gamma^{\mathrm{N}}\right)$ and $\Gamma^{\mathrm{D}}$ is the boundary with a Dirichlet condition $g_{\mathrm{D}}$ such that $g_{\mathrm{D}}$ is a trace on $\Gamma^{\mathrm{D}}$ of a function from $H^{1}(\Omega)$; moreover, we suppose $g_{\mathrm{D}} \in C^{0}\left(\bar{\Gamma}^{\mathrm{D}}\right)$ and, for simplicity, the $(d-1)$-dimensional measure of $\Gamma^{\mathrm{D}}$ nonzero, $\left|\Gamma^{\mathrm{D}}\right|>0$. Other boundary conditions can be treated as well. Furthermore, $f \in L^{2}(\Omega)$ is the source term, $\mathbf{n}$ is the outward unit normal vector to $\partial \Omega$, and $S$ is a symmetric, bounded, and uniformly positive definite tensor whose terms are functions in $L^{\infty}(\Omega)$.

\footnotetext{
${ }^{*}$ This work was supported by ANDRA, the French agency for nuclear waste management, and by the ANR project DEDALES under grant ANR-14-CE23-0005. It has also received funding from the European Research Council (ERC) under the European Union's Horizon 2020 research and innovation program (grant agreement No 647134 GATIPOR).

*Inria Paris, 2 rue Simone Iff, 75589 Paris 12, France \& Université Paris-Est, CERMICS (ENPC), 77455 Marne-la-Vallée 2, France sarah.ali-hassan@inria.fr.

${ }^{\dagger}$ UMR 7539, LAGA, Université Paris 13, 93430 Villetaneuse, France japhet@math.univ-paris13.fr.

${ }^{\ddagger}$ Inria Paris, 2 rue Simone Iff, 75589 Paris 12, France \& Université Paris-Est, CERMICS (ENPC), 77455 Marne-la-Vallée 2, France Michel.Kern@inria.fr, martin.vohralik@inria.fr.
} 
Domain decomposition (DD) methods decompose $\Omega$ into subdomains and then reduce the second order elliptic problem (1.1) to smaller problems on each subdomain. They can be traced back to H. A. Schwarz [47] who used such an idea to prove existence and uniqueness of the solution of Laplace's equation in irregular domains. Then P.-L. Lions [34] introduced a parallelizable nonoverlapping version of the Schwarz method based on Robin transmission conditions. This approach provides a strong basis for domain decomposition methods, in particular for the optimized Schwarz method studied in [27, 28] that is used throughout this paper. This method relies on Robin or Ventcell transmission conditions on the interfaces whose coefficients can be optimized to improve convergence rates. An overview of the optimized Schwarz method is given in $[15,21]$, completed by an extension to a diffusion problem with discontinuous coefficient in [22]. In the context of mixed finite elements, we refer also to $[17,25,26]$. The multi-domain problem can actually be reformulated as an interface problem (see [15] or [25]) that can be solved by various iterative methods, such as block-Jacobi or GMRES.

Several a posteriori error estimates valid during the iteration of an algebraic iterative solver have been derived previously. In particular, Becker, Johnson, and Rannacher in [7] obtain residual-based estimates in the context of conforming finite element discretizations and multigrid solvers. Arioli [4] then derives stopping criteria for the conjugate gradient solver in the same setting, and Arioli and Loghin [5] obtain such results for mixed finite element discretizations. Goal-oriented a posteriori error estimates for linear elliptic problems have also been derived in the inexact solver context, in particular for the primal-dual preconditioned conjugate-gradient Lanczos method by Patera and Rønquist in [38], and for the multigrid algorithm by Meidner, Rannacher, and Vihharev [37]. A general framework taking into account any numerical method and any algebraic solver was then introduced in [18], following some basic ideas of [29], and has since then been used also to coupled unsteady nonlinear and degenerate problems, see $[10,14]$ and the references therein.

Coupling specifically domain decomposition and a posteriori error estimates has also recently been addressed in $[43,44]$. Here, the case of the linear elasticity problem approximated by the finite element method in combination with non-overlapping domain decomposition method such as the finite element tearing and interconnecting (FETI, see [20]) or Balancing Neumann-Neumann (BDD, see [35] and [12] for the case of mixed finite elements) have been studied. The authors derive both upper and lower bounds for the overall error, and the discretization and the domain decomposition error components are distinguished, which leads to an a posteriori stopping criterion. One crucially uses here the nature of the domain decomposition algorithm, where 1) an $H_{0}^{1}(\Omega)$-conforming potential solution is provided at each step, given by the subdomain problems with the Dirichlet condition on the interface; 2) simultaneously, an auxiliary variable with coinciding normal fluxes on the interface results from the subdomain Neumann problems, so that an $\mathbf{H}(\operatorname{div}, \Omega$ )-conforming flux can be easily reconstructed at each step. Then the a posteriori methodology in the spirit of Prager-Synge [40] applies, cf. Ladevèze and Pelle [32], Repin [42], or the recent developments in [19]. This, unfortunately, only seems to be possible in the simultaneous presence of subdomain problems with two types (Dirichlet and Neumann) interface conditions solved at each DD iteration, which is not the case here. To overcome this, our key tools will be, the potential reconstruction $s_{h}^{k}$ of Concept 4.5 and the equilibrated flux reconstruction $\boldsymbol{\sigma}_{h}^{k}$ of Concept 4.6 .

In this contribution, we are interested in general domain decomposition algorithms where on the interfaces, neither the conformity of the flux nor that of the potential is preserved. To exemplify our ideas, we treat (optimized) Schwarz methods with Robin transmission conditions, but any other DD approach can be treated, including Ventcell transmission conditions. We focus on mixed finite element discretizations in the subdomains and extend the approaches from $[2,31,48,49]$ on a posteriori error estimates in mixed methods with exact linear algebra (leading to mass conservation and flux continuity) and in particular the approach from [39] for a posteriori error estimates in mixed methods without flux continuity. We first build a flux reconstruction that is globally $\mathbf{H}(\operatorname{div}, \Omega)$-conforming and locally conservative in each mesh element. In a first stage, a simple coarse balancing problem, with one unknown per interface and two unknowns (in two space dimensions) per each subdomain boundary lying in $\partial \Omega$, is solved. Then we adopt the construction of [39, Section 3.5.2] and solve a local Neumann problem in a band around the interfaces in each subdomain by the mixed finite element method. Finally, two $H^{1}$ (subdomain)-conforming potential reconstructions are built. One is standard relying on the averaging operator $\mathcal{I}_{\text {av }}$ following $[1,8,30]$, whereas the other introduces weights on the interfaces whose goal is to separate the DD and the discretization components.

The outline of the paper is as follows: after introducing some useful notation in Section 2, we present in Section 3 the multi-domain formulation using the optimized Schwarz method and reformulate it as an interface problem. We next show how to solve this interface problem using either a block-Jacobi or a GMRES 
method, and detail the approximation of the corresponding local problems by the mixed finite element method. In Section 4, we derive a fully computable upper bound for the error between the exact and the approximate numerical solutions in an energy norm. The details about the employed flux and potential reconstructions are presented in Section 5. Finally, in Section 6, numerical results for two examples, relying respectively on the block-Jacobi and the GMRES iterations, testify tight overall error control and important reduction of the number of DD iterations.

\section{Preliminaries}

In this section we introduce the partition of $\Omega$ and some function spaces.

\subsection{Partitions of the domain $\Omega$}

We suppose that the domain $\Omega$ is decomposed into $\mathcal{N}$ non-overlapping polygonal subdomains $\Omega_{i}, i \in \llbracket 1, \mathcal{N} \rrbracket$, such that $\bar{\Omega}=\cup_{i=1}^{\mathcal{N}} \bar{\Omega}_{i}$. For all $i \in \llbracket 1, \mathcal{N} \rrbracket$, let $\Gamma_{i}^{\mathrm{N}}:=\Gamma^{\mathrm{N}} \cap \partial \Omega_{i}, \Gamma_{i}^{\mathrm{D}}:=\Gamma^{\mathrm{D}} \cap \partial \Omega_{i}$, and $\mathbf{n}_{i}$ be the unit outward-pointing normal on $\partial \Omega_{i}$. Let $B^{i}$ be the set of neighbors of the subdomain $\Omega_{i}$ that share at least one edge (if $d=2$ ) with $\Omega_{i}$ (face if $d=3$ ) and let $\left|B^{i}\right|$ be the cardinality of this set. Using this notation, we introduce the interface $\Gamma_{i, j}:=\partial \Omega_{i} \cap \partial \Omega_{j}, j \in B^{i}$, between two adjacent subdomains $\Omega_{i}$ and $\Omega_{j}$. Consequently, $\partial \Omega_{i}=\Gamma_{i}^{\mathrm{N}} \cup \Gamma_{i}^{\mathrm{D}} \cup \Gamma_{i}$ with $\Gamma_{i}:=\cup_{j \in B^{i}} \Gamma_{i, j}$. We also define $\Gamma:=\underset{i \in \llbracket 1, \mathcal{N} \rrbracket}{\cup} \Gamma_{i}$.

Define $\mathcal{T}_{h}:=\mathcal{\cup}_{i=1}^{\mathcal{N}} \mathcal{T}_{h, i}$, where $\mathcal{T}_{h, i}$ is a regular triangulation of the subdomain $\Omega_{i}$, such that $\bar{\Omega}_{i}=\underset{K \in \mathcal{T}_{h, i}}{\cup} K$, where $\left|\mathcal{T}_{h, i}\right|$ is the number of triangles (tetrahedra if $d=3$ ) in the $i$-th subdomain. We suppose that $\mathcal{T}_{h, i}$ is a conforming mesh, i.e., such that if $K, K^{\prime} \in \mathcal{T}_{h, i}, K \neq K^{\prime}$, then $K \cap K^{\prime}$ is either an empty set or a common vertex or edge or face. For simplicity, we also assume that $\mathcal{T}_{h}$ is conforming, although this assumption could be easily avoided by introducing the concept of a simplicial submesh as in, e.g., [16, 39] and the references therein. We denote the set of all edges (faces if $d=2$ ) of $\mathcal{T}_{h, i}$ by $\mathcal{E}_{h, i}$, and the set of all edges (faces) of $K$ by $\mathcal{E}_{K}$. Define $\mathcal{E}_{h, i}^{\text {int }}$ to be the set of interior edges (faces) of the subdomain $\Omega_{i}, \mathcal{E}_{h, i}^{\text {ext }}=\mathcal{E}_{h, i}^{\Gamma^{\mathrm{D}}} \cup \mathcal{E}_{h, i}^{\Gamma^{\mathrm{N}}}$ is the set of boundary edges (faces) on $\partial \Omega \cap \partial \Omega_{i}$, and $\mathcal{E}_{h}^{\Gamma_{i, j}}$ is the set of sides on the interfaces $\Gamma_{i, j}$. Then $\mathcal{E}_{h, i}=\left(\cup_{j \in B^{i}} \mathcal{E}_{h}^{\Gamma_{i, j}}\right) \cup \mathcal{E}_{h, i}^{\text {int }} \cup \mathcal{E}_{h, i}^{\text {ext }}$. Let $h_{K}$ denote the diameter of $K$ and let $h_{i}$ be the largest diameter of all triangles (tetrahedra if $d=3$ ) in $\mathcal{T}_{h, i}$, i.e., $h_{i}=\max _{K \in \mathcal{T}_{h, i}} h_{K}$.

\subsection{Some functions spaces}

We recall here the definition of some basic function spaces. For a given nonempty domain $D \subset \Omega$ and a real number $l, 1 \leq l \leq \infty$, we employ the standard functional notations $L^{l}(D)$ and $\mathbf{L}^{l}(D):=\left[L^{l}(D)\right]^{d}$ of Lebesgue spaces. We denote by $(\cdot, \cdot)_{D}$ the scalar product for $L^{2}(D)$ and $\mathbf{L}^{2}(D)$, associated with the norm $\|\cdot\|_{D}$, and by $|D|$ the Lebesgue measure of $D$. Shall $D=\Omega$, the index will be dropped. Let $\langle\cdot, \cdot\rangle_{\gamma}$ be the scalar product for the $d-1$ dimensional $L^{2}(\gamma)$ on $\gamma=\partial D$ or a subset of it. Let also $H^{1}(D):=\left\{v \in L^{2}(D) ; \nabla v \in \mathbf{L}^{2}(D)\right\}$ be the Sobolev space and let $\mathbf{H}(\operatorname{div}, D):=\left\{\mathbf{v} \in \mathbf{L}^{2}(D) ; \nabla \cdot \mathbf{v} \in L^{2}(D)\right\}$ be the space of vector functions whose weak divergence is square integrable.

\section{Multidomain formulation using the optimized Schwarz method}

In this section, we present a nonoverlapping domain decomposition method for solving problem (1.1). For any scalar-, vector-, or tensor-valued function $\varphi$ defined on $\Omega$, let $\varphi_{i}$ denote the restriction of $\varphi$ to $\Omega_{i}, i=1, . ., \mathcal{N}$. By using this notation, problem (1.1) can be reformulated as an equivalent multidomain problem consisting 
of the following subdomain problems (see [33, 41]), for $i \in \llbracket 1, \mathcal{N} \rrbracket$ :

$$
\begin{aligned}
& \mathbf{u}_{i}=-\boldsymbol{S} \nabla p_{i} \quad \text { in } \quad \Omega_{i}, \\
& \nabla \cdot \mathbf{u}_{i}=f \quad \text { in } \Omega_{i}, \\
& p_{i}=g_{\mathrm{D}} \quad \text { on } \quad \Gamma_{i}^{\mathrm{D}}, \\
& -\mathbf{u}_{i} \cdot \mathbf{n}_{i}=g_{\mathrm{N}} \quad \text { on } \quad \Gamma_{i}^{\mathrm{N}},
\end{aligned}
$$

together with the transmission conditions on the interfaces (with $\mathbf{n}_{i}=-\mathbf{n}_{j}$ )

$$
\begin{array}{ccc}
p_{i}=p_{j} & \text { on } & \Gamma_{i, j}, \forall j \in B^{i}, \\
\mathbf{u}_{i} \cdot \mathbf{n}_{i}+\mathbf{u}_{j} \cdot \mathbf{n}_{j}=0 & \text { on } & \Gamma_{i, j}, \forall j \in B^{i} .
\end{array}
$$

Equations (3.1) are the "natural" transmission conditions which ensure the continuity of the pressure head $p$ and of the normal trace of the flux $\mathbf{u}$ on the interface $\Gamma_{i, j}$.

Alternatively and equivalently, see [34], one may impose the Robin transmission conditions

$$
-\beta_{i, j} \mathbf{u}_{i} \cdot \mathbf{n}_{i}+p_{i}=-\beta_{i, j} \mathbf{u}_{j} \cdot \mathbf{n}_{i}+p_{j} \quad \text { on } \quad \Gamma_{i, j}, \forall j \in B^{i}
$$

where $\beta_{i, j}>0, j \in B^{i}, i \in \llbracket 1, \mathcal{N} \rrbracket$ are fixed parameters that may be optimized to improve the convergence rate of the iterative domain decomposition method, see [27, 28] (or [15, 21] for an overview). This method is called the optimized Schwarz method.

Remark 3.1. Note that from (3.2), and by using $\mathbf{n}_{j}=-\mathbf{n}_{i}$, the interface term transmitted from $\Omega_{i}$ to $\Omega_{j}$ will be $\beta_{j, i} \mathbf{u}_{i} \cdot \mathbf{n}_{i}+p_{i}$ on $\Gamma_{i, j}$. Now, in the context of mixed finite elements, $p_{i} \in L^{2}\left(\Omega_{i}\right)$, so that $\left.p_{i}\right|_{\Gamma_{i, j}}$ is not well defined, and must be defined by way of the Robin condition in $\Omega_{i}$. This condition reads $-\beta_{i, j} \mathbf{u}_{i} \cdot \mathbf{n}_{i}+p_{i}=\xi_{i, j}$, with a given Robin boundary data $\xi_{i, j}$ on $\Gamma_{i, j}$, and thus we obtain the well-defined expression

$$
\left.p_{i}\right|_{\Gamma_{i, j}}=\xi_{i, j}+\beta_{i, j} \mathbf{u}_{i} \cdot \mathbf{n}_{i} .
$$

This approach will in particular be used below to define the Robin-to-Robin operator $\mathcal{S}_{i}^{\mathrm{RtR}}$ in (3.6), as well as on the discrete level to define the mixed finite element scheme in Section 3.4.

\subsection{The interface problem}

An interface operator can be used to reformulate the multidomain problem as a problem where the unknowns are located only on the interface (see e.g. [15]). Here the formulation of this interface problem is based on [25] for 2 subdomains, and on [13] for the case of multiple subdomains. Let $\mathcal{V}_{i}:=L^{2}\left(\Omega_{i}\right) \times L^{2}\left(\Gamma_{i}^{\mathrm{D}}\right) \times L^{2}\left(\Gamma_{i}^{\mathrm{N}}\right)$ for $i \in \llbracket 1, \mathcal{N} \rrbracket$. We first introduce the space

$$
\mathbf{W}_{i}:=\left\{\mathbf{v} \in \mathbf{H}\left(\operatorname{div}, \Omega_{i}\right) ; \mathbf{v} \cdot \mathbf{n}_{i} \in L^{2}\left(\partial \Omega_{i}\right)\right\}
$$

with an increased normal trace regularity, as Robin condition will be considered in the sequel. This requirement could possibly be weakened, by proceeding as in the recent article [11], where Dirichlet and Neumann conditions are treated. We, however, only use the space $\mathbf{W}_{i}$ for abstract formulation of the interface problem and for motivation; the present a posteriori error analysis does not rely on it. We then define the sets

$$
\mathbf{W}_{i}^{g_{\mathrm{N}}}:=\left\{\mathbf{v} \in \mathbf{W}_{i} ; \mathbf{v} \cdot \mathbf{n}_{i}=g_{\mathrm{N}} \text { on } \Gamma^{\mathrm{N}} \cap \partial \Omega_{i}\right\}
$$

of functions respecting the Neumann boundary condition on $\Gamma^{\mathrm{N}}$. We now introduce the subproblem solution operator for the subdomain $\Omega_{i}, i \in \llbracket 1, \mathcal{N} \rrbracket$, as follows:

$$
\begin{array}{cccc}
\mathcal{M}_{i}: & L^{2}\left(\Gamma_{i}\right) \times \mathcal{V}_{i} & \rightarrow & L^{2}\left(\Gamma_{i}\right) \times L^{2}\left(\Omega_{i}\right) \times \mathbf{W}_{i}^{g_{\mathrm{N}}} \\
\left(\boldsymbol{\xi}_{i}, \mathcal{F}_{i}\right) & \mapsto & \left(\boldsymbol{\xi}_{i}, p_{i}, \mathbf{u}_{i}\right)
\end{array}
$$


where $L^{2}\left(\Gamma_{i}\right):=\prod_{j \in B^{i}} L^{2}\left(\Gamma_{i, j}\right), \boldsymbol{\xi}_{i}:=\left(\xi_{i, j}\right)_{j \in B^{i}}, \mathcal{F}_{i}:=\left(\left.f\right|_{\Omega_{i}},\left.g_{\mathrm{D}}\right|_{\Gamma_{i}^{\mathrm{D}}},\left.g_{\mathrm{N}}\right|_{\Gamma_{i}^{\mathrm{N}}}\right)$, and where $\left(p_{i}, \mathbf{u}_{i}\right)$ is the solution of the following problem in $\Omega_{i}$ (in an appropriate mixed formulation):

$$
\begin{aligned}
& \mathbf{u}_{i}=-\boldsymbol{S} \nabla p_{i} \quad \text { in } \quad \Omega_{i}, \\
& \nabla \cdot \mathbf{u}_{i}=f \quad \text { in } \Omega_{i}, \\
& p_{i}=g_{\mathrm{D}} \quad \text { on } \quad \Gamma_{i}^{\mathrm{D}}, \\
& -\mathbf{u}_{i} \cdot \mathbf{n}_{i}=g_{\mathrm{N}} \quad \text { on } \Gamma_{i}^{\mathrm{N}}, \\
& -\beta_{i, j} \mathbf{u}_{i} \cdot \mathbf{n}_{i}+p_{i}=\xi_{i, j} \quad \text { on } \quad \Gamma_{i, j}, \forall j \in B^{i} .
\end{aligned}
$$

The operator $\mathcal{M}_{i}$ takes the available Robin condition $\boldsymbol{\xi}_{i}$ and the volume and Dirichlet and Neumann boundary data stored in $\mathcal{F}_{i}$ and maps them to $\boldsymbol{\xi}_{i}$ together with the subdomain pressure head $p_{i}$ and the Darcy velocity $\mathbf{u}_{i}$.

Using Remark 3.1, we also introduce the operator

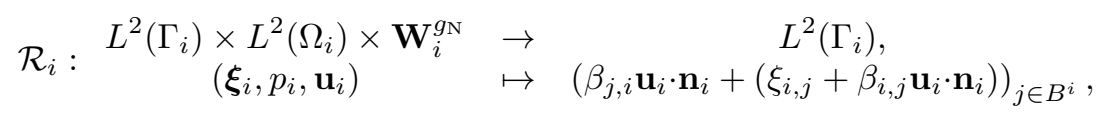

which transforms the available Robin condition $\boldsymbol{\xi}_{i}$ together with the pressure head $p_{i}$ and Darcy velocity $\mathbf{u}_{i}$ to a new Robin datum.

The Robin-to-Robin operator is finally defined as:

$$
\mathcal{S}_{i}^{\mathrm{RtR}}:=\mathcal{R}_{i} \circ \mathcal{M}_{i}: L^{2}\left(\Gamma_{i}\right) \times \mathcal{V}_{i} \rightarrow L^{2}\left(\Gamma_{i}\right) .
$$

Then conditions (3.2) with $\left(p_{i}, \mathbf{u}_{i}\right)$ solution of the subproblem (3.4) lead to the equivalent interface problem: find $\boldsymbol{\xi}:=\left(\boldsymbol{\xi}_{1}, \ldots, \boldsymbol{\xi}_{\mathcal{N}}\right) \in L^{2}(\Gamma):=\prod_{i \in \llbracket 1, \mathcal{N} \rrbracket} L^{2}\left(\Gamma_{i}\right)$ such that

$$
\left(\boldsymbol{\xi}_{i}\right)_{j}=\left(\mathcal{S}_{j}^{\mathrm{RtR}}\left(\boldsymbol{\xi}_{j}, \mathcal{F}_{j}\right)\right)_{i}, \quad \forall j \in B^{i}, \quad \forall i \in \llbracket 1, \mathcal{N} \rrbracket .
$$

Using the fact that $\mathcal{M}_{j}\left(\boldsymbol{\xi}_{j}, \mathcal{F}_{j}\right)=\mathcal{M}_{j}\left(\boldsymbol{\xi}_{j}, \mathbf{0}\right)+\mathcal{M}_{j}\left(\mathbf{0}, \mathcal{F}_{j}\right)$, the linearity of the operator $\mathcal{R}_{i}$, and defining

$$
\begin{array}{clc}
\mathcal{S}_{R}: \quad(\Gamma) & \rightarrow c & L^{2}(\Gamma) \\
\boldsymbol{\xi} & \left.\mapsto\left(\left(\boldsymbol{\xi}_{i}\right)_{j}-\left(\mathcal{S}_{j}^{\mathrm{RtR}}\left(\boldsymbol{\xi}_{j}, \mathbf{0}\right)\right)_{i}\right)_{j \in B^{i}}\right)_{1 \leq i \leq \mathcal{N}},
\end{array}
$$

and

$$
\chi:=\left(\left(\left(\mathcal{S}_{j}^{\mathrm{RtR}}\left(\mathbf{0}, \mathcal{F}_{j}\right)\right)_{i}\right)_{j \in B^{i}}\right)_{1 \leq i \leq \mathcal{N}},
$$

problem (3.7) can be rewritten as:

$$
\mathcal{S}_{R} \boldsymbol{\xi}=\chi
$$

The interface problem (3.9) is usually solved by iterative methods, using block-Jacobi iterations or GMRES.

\subsection{Solving the interface problem by the block-Jacobi method}

The simplest method for solving the interface problem (3.9) is a block-Jacobi method (equivalent to Richardson's iteration in our case, because the "diagonal" of the operator is zero). To show the similarity with the GMRES method introduced below, we write the algorithm as follows: given an initial guess $\xi^{0} \in L^{2}(\Gamma)$, at iteration $k \geq 1$ compute the residual

$$
\boldsymbol{r}^{k-1}:=\chi-\mathcal{S}_{R} \boldsymbol{\xi}^{k-1}
$$

and define a new iterate by

$$
\boldsymbol{\xi}^{k}:=\boldsymbol{\xi}^{k-1}+\boldsymbol{r}^{k-1}
$$


The block-Jacobi algorithm applied to the interface problem (3.9) is equivalent to solving local subdomain problems and then transferring information to the neighboring subdomain. At each iteration $k \geq 1$ of this algorithm, for $i \in \llbracket 1, \mathcal{N} \rrbracket$, one needs to find $p_{i}^{k}$ and $\mathbf{u}_{i}^{k}$ in subdomain $\Omega_{i}$ such that:

$$
\begin{aligned}
\mathbf{u}_{i}^{k} & =-\boldsymbol{S} \nabla p_{i}^{k} & & \text { in } \Omega_{i}, \\
\nabla \cdot \mathbf{u}_{i}^{k} & =f & & \text { in } \Omega_{i}, \\
p_{i}^{k} & =g_{\mathrm{D}} & & \text { on } \Gamma_{i}^{\mathrm{D}}, \\
-\mathbf{u}_{i}^{k} \cdot \mathbf{n}_{i} & =g_{\mathrm{N}} & & \text { on } \Gamma_{i}^{\mathrm{N}}, \\
-\beta_{i, j} \mathbf{u}_{i}^{k} \cdot \mathbf{n}_{i}+p_{i}^{k} & =\xi_{i, j}^{k-1} & & \text { on } \Gamma_{i, j}, \forall j \in B^{i},
\end{aligned}
$$

where $\xi_{i, j}^{k-1}:=-\beta_{i, j} \mathbf{u}_{j}^{k-1} \cdot \mathbf{n}_{i}+p_{j}^{k-1}$ is the information coming from the neighboring subdomain $\Omega_{j}, j \in B^{i}$, at step $k-1$ of the algorithm. The initial guess $\boldsymbol{\xi}^{0} \in L^{2}(\Gamma)$ is a given function in $L^{2}\left(\Gamma_{i, j}\right)$. The convergence analysis of this algorithm has been carried out in [17].

Remark 3.2. Note that the continuity of the normal traces $\mathbf{u}_{i} \cdot \mathbf{n}_{i}=\mathbf{u}_{j} \cdot \mathbf{n}_{i}$ and of the pressure $p_{i}=p_{j}$ will be satisfied only at convergence of the DD algorithm.

\subsection{Solving the interface problem by the GMRES method}

To obtain faster convergence, one can use Krylov acceleration techniques for solving the interface problem, such as GMRES [45, 46]. For this purpose, let us consider the interface problem (3.9). Given an initial guess $\boldsymbol{\xi}^{0} \in L^{2}(\Gamma)$ and the corresponding residual $\boldsymbol{r}^{0}:=\chi-\mathcal{S}_{R} \boldsymbol{\xi}^{0} \in L^{2}(\Gamma)$, let

$$
\mathcal{K}_{k}:=\mathcal{K}_{k}\left(\mathcal{S}_{R}, \boldsymbol{r}^{0}\right):=\operatorname{span}\left\{\boldsymbol{r}^{0}, \mathcal{S}_{R} \boldsymbol{r}^{0}, \mathcal{S}_{R}^{2} \boldsymbol{r}^{0}, \ldots, \mathcal{S}_{R}^{k-1} \boldsymbol{r}^{0}\right\} \subset L^{2}(\Gamma)
$$

be the $k$-th Krylov subspace for this problem, $k \geq 1$. Note that the iterates for the block-Jacobi method introduced above all belong to the space $\boldsymbol{\xi}^{0}+\mathcal{K}_{k}$. The GMRES algorithm (see e.g. [9, 23] for infinitedimensional spaces) generates a sequence of iterates $\left\{\boldsymbol{\xi}^{k}\right\}_{k \geq 1}$, where $\boldsymbol{\xi}^{k}$ is a solution of the finite-dimensional minimization problem

$$
\min _{\boldsymbol{\xi} \in \boldsymbol{\xi}^{0}+\mathcal{K}_{k}}\left\|\mathcal{S}_{R} \boldsymbol{\xi}-\chi\right\|_{L^{2}(\Gamma)}
$$

Let $\left\{\mathrm{e}_{1}, \ldots, \mathrm{e}_{k}\right\}$ denote the vectors of the canonical basis of $\mathbb{R}^{k}$. At the $k$-th GMRES iteration, $k \geq 1$, the calculation of $\boldsymbol{\xi}^{k}$ requires the computation of functions $\boldsymbol{q}_{1}, \ldots, \boldsymbol{q}_{k+1} \in L^{2}(\Gamma)$ that form an orthonormal basis of $\mathcal{K}_{k+1}$ using the Arnoldi method. More precisely, the Arnoldi algorithm computes a matrix $H_{k} \in \mathbb{R}^{k \times k}$ and an element $\boldsymbol{f}_{k+1} \in L^{2}(\Gamma)$ such that

$$
\mathcal{S}_{R} Q_{k} \mathrm{y}=Q_{k} H_{k} \mathrm{y}+\boldsymbol{f}_{k+1} \mathrm{e}_{\mathrm{k}}^{T} \mathrm{y} \quad \forall \mathrm{y} \in \mathbb{R}^{k},
$$

where the operator $Q_{k}: \mathbb{R}^{k} \rightarrow \mathcal{K}_{k}$ is defined by $Q_{k} \mathrm{y}=\sum_{j=1}^{k}\left(\mathrm{e}_{j}^{T} \mathrm{y}\right) \boldsymbol{q}_{j}, \mathrm{y} \in \mathbb{R}^{k}$. If $\boldsymbol{f}_{k+1} \neq 0$, equation (3.11) is rewritten as

$$
\mathcal{S}_{R} Q_{k} \mathrm{y}=Q_{k+1} \bar{H}_{k} \mathrm{y} \quad \forall \mathrm{y} \in \mathbb{R}^{k},
$$

where $\bar{H}_{k}$ is the matrix in $\mathbb{R}^{(k+1) \times k}$ obtained by appending to $H_{k}$ the row $\left\|\boldsymbol{f}_{k+1}\right\|_{L^{2}(\Gamma)} \mathrm{e}_{\mathrm{k}}^{T}$, and where the operator $Q_{k+1}$ is defined as $Q_{k}$, replacing $k$ by $k+1$ and with $\boldsymbol{q}_{k+1}:=\boldsymbol{f}_{k+1} /\left\|\boldsymbol{f}_{k+1}\right\|_{L^{2}(\Gamma)}$. Then problem (3.10) can be rewritten as

$$
\min _{\boldsymbol{\xi} \in \boldsymbol{\xi}^{0}+\mathcal{K}_{k}}\left\|\mathcal{S}_{R} \boldsymbol{\xi}-\chi\right\|_{L^{2}(\Gamma)}=\min _{\mathrm{y} \in \mathbb{R}^{k}}\|\| \boldsymbol{r}^{0}\left\|_{L^{2}(\Gamma)} \mathrm{e}_{1}-\bar{H}_{k} \mathrm{y}\right\|_{2}
$$

where $\|\cdot\|_{2}$ is the $l_{2}$-norm on $\mathbb{R}^{k}$. The $k$-th GMRES iteration is then as follows:

1. Compute an orthonormal basis $\left\{\boldsymbol{q}_{1}, \ldots, \boldsymbol{q}_{k}\right\}$ of $\mathcal{K}_{k}$ using the Arnoldi method.

2. Find the $\mathrm{y}^{k}$ which solves the unconstrained (full rank) least squares problem in (3.12).

3. Compute $\boldsymbol{\xi}^{k}:=\boldsymbol{\xi}^{0}+Q_{k} \mathrm{y}^{k}$. 
One repeats the iteration in $k$ until the residual \|\| $\boldsymbol{r}^{0}\left\|_{L^{2}(\Gamma)} \mathrm{e}_{1}-\bar{H}_{k} \mathrm{y}^{k}\right\|_{2}$ becomes small enough. Note that if $\boldsymbol{f}_{k+1}=0$ then $\boldsymbol{\xi}^{k}$ is the exact solution to our problem.

At each iteration, the action of the operator $\mathcal{S}_{R}$ on the vectors $\boldsymbol{q}_{k}$ must be calculated. It uses definition (3.8) and thus involves solving the local subdomain problem in the form (3.4) (in approprate mixed formulation and replacing $\boldsymbol{\xi}$ by $\boldsymbol{q}^{k}$ in $\left.(3.4 \mathrm{e})\right)$.

\subsection{Approximation of the subdomain problems by the mixed finite element method}

We now introduce the discrete counterparts of the block-Jacobi or GMRES algorithms introduced in Sections 3.2 and 3.3, respectively. They consist in using the mixed finite element method to approximate the subdomain problems (3.4). For both methods, the main ingredient is thus the computation of the action of the discrete Robin-to-Robin interface operator to an arbitrary argument that becomes an algebraic vector $\boldsymbol{\xi}$. We now show how this computation can be realized.

Let $M_{h, i} \times \mathbf{W}_{h, i} \subset L^{2}\left(\Omega_{i}\right) \times \mathbf{H}\left(\operatorname{div}, \Omega_{i}\right)$ be the Raviart-Thomas-Nédélec mixed finite element spaces of order 0 for each subdomain $\Omega_{i}$. Here,

$$
M_{h, i}:=\left\{q_{h, i} \in L^{2}\left(\Omega_{i}\right) ;\left.q_{h, i}\right|_{K} \in \mathbb{P}_{0}(K) \quad \forall K \in \mathcal{T}_{h, i}\right\},
$$

where $\mathbb{P}_{0}(K)$ is the space of polynomials of degree 0 , and

$$
\mathbf{W}_{h, i}:=\left\{\mathbf{v}_{h, i} \in \mathbf{H}\left(\operatorname{div}, \Omega_{i}\right) ;\left.\mathbf{v}_{h, i}\right|_{K} \in \mathbf{R T N}_{0}(K) \quad \forall K \in \mathcal{T}_{h, i}\right\},
$$

where $\mathbf{R T N}_{0}(K):=\left[\mathbb{P}_{0}(K)\right]^{d}+\mathbf{x} \mathbb{P}_{0}(K), \mathbf{x} \in \mathbb{R}^{d}$, is the Raviart-Thomas-Nédélec vectorial field space of degree zero defined locally over an element $K \in \mathcal{T}_{h, i}$. We also define the approximation $g_{h, \mathrm{~N}}$ of the function $g_{\mathrm{N}}$ as a piecewise constant function on each edge (face if $d=3$ ) $e \subset \Gamma^{\mathrm{N}}$,

$$
\left.g_{h, \mathrm{~N}}\right|_{e}:=\frac{1}{|e|} \int_{e} g_{\mathrm{N}} \mathrm{d} \gamma,
$$

where $|e|$ is the measure of $e$. We then define the following set:

$$
\mathbf{W}_{h, i}^{g_{h, \mathrm{~N}}}:=\left\{\mathbf{w}_{h, i} \in \mathbf{W}_{h, i} ; \mathbf{w}_{h, i} \cdot \mathbf{n}=g_{h, \mathrm{~N}} \text { on } \Gamma_{i}^{\mathrm{N}}\right\} .
$$

Let $\boldsymbol{\xi}_{h}:=\left(\boldsymbol{\xi}_{h, 1}, \ldots, \boldsymbol{\xi}_{h, \mathcal{N}}\right)$, where $\boldsymbol{\xi}_{h, i}$ is piecewise constant on $\underset{j \in B^{i}}{\cup} \mathcal{E}_{h}^{\Gamma_{i, j}}$ with the values $\xi_{h, i, j}$; this is the discrete Robin condition. The discrete formulation of problem (3.4) can then be written as: find $\mathbf{u}_{h, i} \in \mathbf{W}_{h, i}^{g_{h, N}}$ and $p_{h, i} \in M_{h, i}$ such that:

$$
\begin{aligned}
\mathbf{a}_{i}\left(\mathbf{u}_{h, i}, \mathbf{v}_{h, i}\right)-\mathbf{b}_{i}\left(\mathbf{v}_{h, i}, p_{h, i}\right) & =\boldsymbol{\ell}_{i}\left(\mathbf{v}_{h, i}\right), & & \forall \mathbf{v}_{h, i} \in \mathbf{W}_{h, i}^{0}, \\
\mathbf{b}_{i}\left(\mathbf{u}_{h, i}, q_{h, i}\right) & =\left(f, q_{h, i}\right)_{\Omega_{i}}, & & \forall q_{h, i} \in M_{h, i} .
\end{aligned}
$$

We define the approximate solution $\left(p_{h}, \mathbf{u}_{h}\right)$ such that:

$$
\left.p_{h}\right|_{\Omega_{i}}:=p_{h, i},\left.\quad \mathbf{u}_{h}\right|_{\Omega_{i}}:=\mathbf{u}_{h, i}, \forall i \in \llbracket 1, \mathcal{N} \rrbracket .
$$

The bilinear forms $\mathbf{a}_{i}$ and $\mathbf{b}_{i}$, and the linear form $\boldsymbol{\ell}_{i}$, are as follows:

$$
\begin{array}{rlrl}
\mathbf{a}_{i}: \mathbf{W}_{h, i} \times \mathbf{W}_{h, i} & \longmapsto \mathbb{R}, \quad \mathbf{a}_{i}(\mathbf{u}, \mathbf{v}) & =\left(\boldsymbol{S}^{-1} \mathbf{u}, \mathbf{v}\right)_{\Omega_{i}}+\sum_{j \in B^{i}}\left\langle\beta_{i, j} \mathbf{u} \cdot \mathbf{n}_{i}, \mathbf{v} \cdot \mathbf{n}_{i}\right\rangle_{\Gamma_{i, j}}, \\
\mathbf{b}_{i}: \mathbf{W}_{h, i} \times M_{h, i} & \longmapsto \mathbb{R}, \quad \mathbf{b}_{i}(\mathbf{v}, p)=(p, \nabla \cdot \mathbf{v})_{\Omega_{i}}, \\
\boldsymbol{\ell}_{i}: \quad \mathbf{W}_{h, i} & \longmapsto \mathbb{R}, \quad \boldsymbol{\ell}_{i}(\mathbf{v})=-\left\langle g_{\mathrm{D}}, \mathbf{v} \cdot \mathbf{n}_{i}\right\rangle_{\Gamma_{i}^{\mathrm{D}}}-\sum_{j \in B^{i}}\left\langle\xi_{h, i, j}, \mathbf{v} \cdot \mathbf{n}_{i}\right\rangle_{\Gamma_{i, j}} .
\end{array}
$$

This thus defines a discrete version of the operator $\mathcal{M}_{i}$ from (3.3), where in particular we keep the same definition of the datum $\mathcal{F}_{i}$, with only $g_{h, \mathrm{~N}}$ in place of $g_{\mathrm{N}}$. Proceeding similarly for the operator $\mathcal{R}_{i}$ of $(3.5)$, the discrete version of the Robin-to-Robin interface operator $\mathcal{S}_{i}^{\mathrm{RtR}}$ from (3.6) is, for $i \in \llbracket 1, \mathcal{N} \rrbracket$,

$$
\mathcal{S}_{h, i}^{\mathrm{RtR}}\left(\boldsymbol{\xi}_{h, i}, \mathcal{F}_{i}\right)=\left(\beta_{j, i} \mathbf{u}_{h, i} \cdot \mathbf{n}_{i}+\xi_{h, i, j}+\beta_{i, j} \mathbf{u}_{h, i} \cdot \mathbf{n}_{i}\right)_{j \in B^{i}} .
$$


The discrete interface problem is now defined as in (3.8)-(3.9). Applying the block-Jacobi iteration from Section 3.2 or the GMRES iteration from Section 3.3 gives rise to the discrete approximations $p_{h, i}^{k}$ and $\mathbf{u}_{h, i}^{k}$ and their global counterparts

$$
\left.p_{h}^{k}\right|_{\Omega_{i}}:=p_{h, i}^{k},\left.\quad \mathbf{u}_{h}^{k}\right|_{\Omega_{i}}:=\mathbf{u}_{h, i}^{k}, \forall i \in \llbracket 1, \mathcal{N} \rrbracket .
$$

Remark 3.3. As noticed above in Remark 3.2, there is a continuity of the normal traces of $\mathbf{u}_{h}^{k}$ across the sides between two simplices in each subdomain $\Omega_{i}$ but not across the interfaces in $\Gamma_{i}$ at each iteration of the DD algorithm. The continuity of the normal traces of $\mathbf{u}_{h}^{k}$ (and the pressure in the sense of Remark 4.3 below) will only be satisfied at convergence of the DD algorithm.

\section{A posteriori error estimates}

The purpose of this section is to bound the error between the weak solution of (1.1) and the approximate solution obtained at step $k \geq 1$ of the domain decomposition iteration with mixed finite element discretization (3.13) by indicators that are completely computable from the approximate solution $\left(p_{h}^{k}, \mathbf{u}_{h}^{k}\right)$. We define a suitable postprocessing of the pressure in Section 4.1, introduce the concepts of $H^{1}$ - and $\mathbf{H}(\operatorname{div}, \Omega)$-conforming reconstructions in Section 4.2, and derive the estimates in Section 4.3. Details of the reconstructions will be treated later in Section 5.

We suppose henceforth for simplicity that $g_{\mathrm{D}} \in \mathbb{P}_{2}\left(\cup_{i=1}^{\mathcal{N}} \mathcal{E}_{h, i}^{\Gamma^{\mathrm{D}}}\right) \cap C^{0}\left(\Gamma^{\mathrm{D}}\right)$ and $g_{\mathrm{N}} \in \mathbb{P}_{0}\left(\cup_{i=1}^{\mathcal{N}} \mathcal{E}_{h, i}^{\Gamma^{\mathrm{N}}}\right)$ are respectively piecewise polynomials of total degree less than or equal to 2 and 0 on the Dirichlet and Neumann boundaries. We introduce the broken Sobolev space

$$
H^{1}\left(\mathcal{T}_{h}\right):=\left\{v \in L^{2}(\Omega) ;\left.v\right|_{K} \in H^{1}(K), \quad \forall K \in \mathcal{T}_{h}\right\}
$$

For each interior edge (face if $d=3) e \in\left(\cup_{j \in B^{i}} \mathcal{E}_{h}^{\Gamma_{i, j}}\right) \cup \mathcal{E}_{h, i}^{\text {int }}$ such that the simplices $K$ and $K^{\prime}$ share $e$ (the order of $K, K^{\prime}$ is arbitrary but fixed once and for all), we denote by $\mathbf{n}_{e}$ the normal vector pointing from $K$ to $K^{\prime}$. For a given function $v$, its jump and average are then defined respectively as:

$$
\begin{cases}\llbracket v \rrbracket:=\left.v\right|_{K}-\left.v\right|_{K^{\prime}} \text { and }\left\{\{v\}:=\frac{1}{2}\left(\left.v\right|_{K}+\left.v\right|_{K^{\prime}}\right)\right. & \text { if } e \in\left(\underset{j \in B^{i}}{\cup} \mathcal{E}_{h}^{\Gamma_{i, j}}\right) \cup \mathcal{E}_{h, i}^{\text {int }} \\ \llbracket v \rrbracket:=\left.v\right|_{e}-g_{\mathrm{D}} \text { and }\left\{v v \rrbracket:=\frac{1}{2}\left(\left.v\right|_{e}+g_{\mathrm{D}}\right)\right. & \text { if } e \in \mathcal{E}_{h, i}^{\Gamma^{\mathrm{D}}} .\end{cases}
$$

We define the energy semi-norm on $H^{1}\left(\mathcal{T}_{h}\right)$ by

$$
\|\varphi\|^{2}:=\sum_{K \in \mathcal{T}_{h}}\|\varphi\|_{K}^{2}:=\sum_{K \in \mathcal{T}_{h}}\left\|S^{\frac{1}{2}} \nabla \varphi\right\|_{K}^{2} \quad \varphi \in H^{1}\left(\mathcal{T}_{h}\right)
$$

and the energy norm on $\mathbf{L}^{2}(\Omega)$ by

$$
\|\mathbf{v}\|\left\|_{\star}^{2}:=\sum_{K \in \mathcal{T}_{h}}\right\| \mathbf{v}\left\|_{\star, K}^{2}:=\sum_{K \in \mathcal{T}_{h}}\right\| \boldsymbol{S}^{-\frac{1}{2}} \mathbf{v} \|_{K}^{2} \quad \mathbf{v} \in \mathbf{L}^{2}(\Omega) .
$$

\subsection{Postprocessing of the approximate solution}

Following $[3,6,48]$, we first construct a postprocessing $\tilde{p}_{h, i}^{k}$ of $p_{h, i}^{k}, i \in \llbracket 1, \mathcal{N} \rrbracket$, at each iteration $k \geq 1$ of the DD algorithm. This postprocessing is more regular (piecewise polynomial of total degree less than or equal to 2 on each element that we denote by $\left.\mathbb{P}_{2}\left(\mathcal{T}_{h, i}\right)\right)$ than the piecewise constant $p_{h}^{k}$, so that an application of the piecewise gradient in the energy norm becomes reasonable.

Definition 4.1 (Postprocessing of $\left.p_{h}^{k}\right)$. Construct $\tilde{p}_{h, i}^{k} \in \mathbb{P}_{2}\left(\mathcal{T}_{h, i}\right)$, for all $i \in \llbracket 1, \mathcal{N} \rrbracket$, such that

$$
\begin{aligned}
-\left.\boldsymbol{S} \nabla \tilde{p}_{h, i}^{k}\right|_{K} & =\left.\mathbf{u}_{h, i}^{k}\right|_{K}, & & \forall K \in \mathcal{T}_{h, i}, \\
\left(\tilde{p}_{h, i}^{k}, 1\right)_{K} & =\left(p_{h, i}^{k}, 1\right)_{K}, & & \forall K \in \mathcal{T}_{h, i} .
\end{aligned}
$$


Remark 4.2. The postprocessing $\tilde{p}_{h, i}^{k}$ does not lie in the space $H^{1}\left(\Omega_{i}\right)$, but it follows easily from (3.13a), cf. [48], that $\tilde{p}_{h, i}^{k}$ is weakly continuous,

$$
\left\langle\llbracket \tilde{p}_{h, i}^{k} \rrbracket, 1\right\rangle_{e}=0 \text { for all } e \in \mathcal{E}_{h, i}^{\mathrm{int}}
$$

Similarly, on Dirichlet edges (faces) $e \in \mathcal{E}_{h, i}^{\Gamma^{\mathrm{D}}}$,

$$
\left\langle\tilde{p}_{h, i}^{k}, 1\right\rangle_{e}=\left\langle g_{\mathrm{D}}, 1\right\rangle_{e} .
$$

Remark 4.3. For $j \in B^{i}, \tilde{p}_{h, i}^{k}$ and $\tilde{p}_{h, j}^{k}$ are constructed separately and independently in the two subdomains $\Omega_{i}$ and $\Omega_{j}$. Hence, similarly to Remark 3.2, $\left\langle\llbracket \tilde{p}_{h}^{k} \rrbracket, 1\right\rangle_{e}=0$ for $e \in \mathcal{E}_{h}^{\Gamma_{i, j}}$ only holds at convergence.

\subsection{Concept of $H^{1}$ - and $\mathrm{H}(\operatorname{div}, \Omega)$-conforming reconstructions}

We introduce here the concepts of reconstructions needed in our a posteriori analysis; concrete formulas are given in Section 5. On iteration $k \geq 1$, we construct, in extension of $[39,49]$, three auxiliary objects $\bar{s}_{h}^{k}, s_{h}^{k}$, and $\boldsymbol{\sigma}_{h}^{k}$ :

Concept 4.4 (Subdomain potential reconstructions). We will call a subdomain potential reconstruction, for $\Omega_{i}, i \in \llbracket 1, \mathcal{N} \rrbracket$, any function $\bar{s}_{h, i}^{k}$ constructed from $\tilde{p}_{h, i}^{k}, \mathbf{u}_{h, i}^{k}$ such that

- it is subdomain $H^{1}\left(\Omega_{i}\right)$-conforming, i.e.,

$$
\bar{s}_{h, i}^{k} \in H^{1}\left(\Omega_{i}\right) \cap C^{0}\left(\bar{\Omega}_{i}\right),\left.\quad \bar{s}_{h, i}^{k}\right|_{\Gamma_{i}^{\mathrm{D}}}=\left.g_{\mathrm{D}}\right|_{\Gamma_{i}^{\mathrm{D}}} ;
$$

- it is built locally on each subdomain $\Omega_{i}$ and it should discard as much as possible the influence of the domain decomposition error;

- the comparison of the flux given by this function with $\mathbf{u}_{h, i}^{k}$ estimates the discretization error in each subdomain.

We set as usual $\left.\bar{s}_{h}^{k}\right|_{\Omega_{i}}:=\bar{s}_{h, i}^{k}$.

Concept 4.5 (Potential reconstruction). We call a potential reconstruction any function $s_{h}^{k}$ constructed from $\tilde{p}_{h}^{k}$ such that

- it is globally $H^{1}(\Omega)$-conforming, i.e.,

$$
s_{h}^{k} \in H^{1}(\Omega) \cap C^{0}(\bar{\Omega}),\left.\quad s_{h}^{k}\right|_{\Gamma^{\mathrm{D}}}=g_{\mathrm{D}} ;
$$

- its comparison with $\bar{s}_{h, i}^{k}$ estimates the domain decomposition error in the sense that $\left\|\left|\boldsymbol{S} \nabla\left(\bar{s}_{h}^{k}-s_{h}^{k}\right)\right|\right\|_{\star} \rightarrow 0$ when $k \rightarrow \infty$.

Concept 4.6 (Equilibrated flux reconstruction). We will call an equilibrated flux reconstruction any function $\boldsymbol{\sigma}_{h}^{k}$ constructed from $p_{h}^{k}, \mathbf{u}_{h}^{k}$ such that

- it is $\mathbf{H}(\operatorname{div}, \Omega)$-conforming and locally conservative on the mesh $\mathcal{T}_{h}$, i.e.,

$$
\begin{gathered}
\boldsymbol{\sigma}_{h}^{k} \in \mathbf{H}(\operatorname{div}, \Omega), \\
\left(\nabla \cdot \boldsymbol{\sigma}_{h}^{k}, 1\right)_{K}=(f, 1)_{K}, \quad \forall K \in \mathcal{T}_{h}, \\
-\left(\boldsymbol{\sigma}_{h}^{k} \cdot \mathbf{n}, 1\right)_{e}=\left(g_{\mathrm{N}}, 1\right)_{e}, \quad \forall e \in \mathcal{\mathcal { N }}_{i=1} \mathcal{E}_{h, i}^{\Gamma^{\mathrm{N}}} ;
\end{gathered}
$$

- its comparison with $\mathbf{u}_{h}^{k}$ can be used to estimate the DD error in the sense that $\left\|\left|\mathbf{u}_{h}^{k}-\boldsymbol{\sigma}_{h}^{k}\right|\right\|_{\star} \rightarrow 0$ when $k \rightarrow \infty$. 


\subsection{General a posteriori error estimates for $\tilde{p}_{h} \in H^{1}\left(\mathcal{T}_{h}\right)$ and $\mathbf{u}_{h} \in \mathbf{L}^{2}(\Omega)$}

In this section, we present a general form of our a posteriori error estimates, independent of the discretization method used in each subdomain and based on the results given in [49] and [39]. Our main result bounds both the error due to the discretization in the subdomains and the error due to the domain decomposition iterations:

Theorem 4.7 (A posteriori error estimates for the flux). Let $\mathbf{u} \in \mathbf{H}(\operatorname{div}, \Omega)$ be the weak solution of the problem (1.1) and let $\mathbf{u}_{h}^{k} \in \mathbf{L}^{2}(\Omega)$ be an arbitrary approximation, in particular $\mathbf{u}_{h}^{k}$ can be the solution of the discrete problem (3.13) at iteration $k$ of a DD iterative algorithm (block-Jacobi, GMRES, or other). Let $\bar{s}_{h}^{k}$ be the subdomain potential reconstruction of Concept $4.4, s_{h}^{k}$ the potential reconstruction of Concept 4.5, and $\boldsymbol{\sigma}_{h}^{k}$ the equilibrated flux reconstruction of Concept 4.6. Then the following bound holds:

$$
\begin{aligned}
\left\|\mid \mathbf{u}-\mathbf{u}_{h}^{k}\right\| \|_{\star} \leq \eta^{k}:= & \overbrace{\left\{\sum_{K \in \mathcal{T}_{h}}\left(\eta_{\mathrm{CR}, K}^{k}\right)^{2}\right\}^{\frac{1}{2}}+\left\{\sum_{K \in \mathcal{T}_{h}}\left(\eta_{\mathrm{osc}, K}^{k}\right)^{2}\right\}^{\frac{1}{2}}}^{:=\eta_{\mathrm{disc}, \mathbf{u}}^{k}} \\
& +\underbrace{\left\{\sum_{K \in \mathcal{T}_{h}}\left(\eta_{\mathrm{DDF}, K}^{k}\right)^{2}\right\}^{\frac{1}{2}}+\left\{\sum_{K \in \mathcal{T}_{h}}\left(\eta_{\mathrm{DDP}, K}^{k}\right)^{2}\right\}^{\frac{1}{2}}}_{:=\eta_{\mathrm{DD}}^{k}},
\end{aligned}
$$

where

$$
\begin{aligned}
\eta_{\mathrm{CR}, K}^{k} & :=\left\|\mid \mathbf{u}_{h}^{k}+\boldsymbol{S} \nabla \bar{s}_{h}^{k}\right\| \|_{\star, K}, & & \text { constitutive relation, } \\
\eta_{\mathrm{DDP}, K}^{k} & :=\left\||| \boldsymbol{S} \nabla\left(\bar{s}_{h}^{k}-s_{h}^{k}\right)\right\| \|_{\star, K}, & & \text { DD potential nonconformity, } \\
\eta_{\mathrm{DDF}, K}^{k} & :=\left.\left\||| \mathbf{u}_{h}^{k}-\boldsymbol{\sigma}_{h}^{k}\right\|\right|_{\star, K}, & & \text { DD flux nonconformity, } \\
\eta_{\mathrm{osc}, K}^{k} & :=\frac{h_{K}}{\pi} c_{\boldsymbol{S}, K}^{-\frac{1}{2}}\left\|f-\nabla \cdot \boldsymbol{\sigma}_{h}^{k}\right\|_{K}, & & \text { data oscillation. }
\end{aligned}
$$

Here $c_{\boldsymbol{S}, K}$ is the smallest eigenvalue of the tensor $\boldsymbol{S}$ in $K$. The discretization error estimator (also called subdomain estimator) is denoted by $\eta_{\mathrm{disc}, \mathbf{u}}^{k}$ and the domain decomposition estimator (the interface estimator) is denoted by $\eta_{\mathrm{DD}}^{k}$.

Proof. It follows readily from Therorem 3.1 in [39] that for the DD method where the flux and the potential are not continuous on the interface, we have

$$
\begin{aligned}
\left\|\mid \mathbf{u}-\mathbf{u}_{h}^{k}\right\| \|_{\star} \leq & \left\{\sum_{K \in \mathcal{T}_{h}}\left\|\mathbf{u}_{h}^{k}+S \nabla s_{h}^{k} \mid\right\|_{\star, K}^{2}\right\}^{\frac{1}{2}}+\left\{\sum_{K \in \mathcal{T}_{h}}\left(\eta_{\mathrm{DDF}, K}^{k}\right)^{2}\right\}^{\frac{1}{2}} \\
& +\left\{\sum_{K \in \mathcal{T}_{h}}\left(\eta_{\mathrm{osc}, K}^{k}\right)^{2}\right\}^{\frac{1}{2}} .
\end{aligned}
$$

The triangle inequality on the space $l^{2}$ on $\mathbb{R}^{\left|\mathcal{T}_{h}\right|}$ then completes the proof:

$$
\left\{\sum_{K \in \mathcal{T}_{h}}\left|\left\|\mathbf{u}_{h}^{k}+\boldsymbol{S} \nabla s_{h}^{k} \mid\right\|_{\star, K}^{2}\right\}^{\frac{1}{2}} \leq\left\{\sum_{K \in \mathcal{T}_{h}}\left(\eta_{\mathrm{CR}, K}^{k}\right)^{2}\right\}^{\frac{1}{2}}+\left\{\sum_{K \in \mathcal{T}_{h}}\left(\eta_{\mathrm{DDP}, K}^{k}\right)^{2}\right\}^{\frac{1}{2}} .\right.
$$

Similarly, [39, Therorem 3.1] readily yields an estimate for the potential:

Corollary 4.8 (A posteriori error estimates for the potential). Let $p$ be the weak solution of the problem (1.1) and let $\tilde{p}_{h}^{k} \in H^{1}\left(\mathcal{T}_{h}\right)$ be an arbitrary approximation, in particular $\tilde{p}_{h}^{k}$ can be the postprocessing of $p_{h}^{k}$ solution 
of problem (3.13) at iteration $k$ of a DD iterative algorithm given by Definition 4.1. Let $\mathbf{u}_{h}^{k}=-\boldsymbol{S} \nabla \tilde{p}_{h}^{k}$. Let $\bar{s}_{h}^{k}, s_{h}^{k}$, and $\boldsymbol{\sigma}_{h}^{k}$ be respectively given by Concepts 4.4, 4.5, and 4.6. Then the following bound holds:

$$
\begin{aligned}
&\left\|\left|p-\tilde{p}_{h}^{k} \|\right| \leq \tilde{\eta}^{k}:=\right. \overbrace{\left\{\sum_{K \in \mathcal{T}_{h}}\left(\eta_{\mathrm{NCP}, K}^{k}\right)^{2}\right\}^{\frac{1}{2}}+\left\{\sum_{K \in \mathcal{T}_{h}}\left(\eta_{\mathrm{osc}, K}^{k}\right)^{2}\right\}^{\frac{1}{2}}}^{\eta_{\mathrm{disc}, p}^{k}} \\
&+\underbrace{}_{\left\{\sum_{\mathrm{D} \in \mathcal{T}_{h}}\left(\eta_{\mathrm{DDF}, K}^{k}\right)^{2}\right\}^{\frac{1}{2}}+\left\{\sum_{K \in \mathcal{T}_{h}}\left(\eta_{\mathrm{DDP}, K}^{k}\right)^{2}\right\}^{\frac{1}{2}}},
\end{aligned}
$$

where the potential nonconformity estimator $\eta_{\mathrm{NCP}, K}^{k}$ is given by

$$
\eta_{\mathrm{NCP}, K}^{k}:=|| \tilde{p}_{h}^{k}-\bar{s}_{h}^{k} \mid \|_{K}
$$

and $\eta_{\mathrm{DDP}, K}^{k}, \eta_{\mathrm{DDF}, K}^{k}$, and $\eta_{\mathrm{osc}, K}^{k}$ are respectively given by $(4.2 \mathrm{~b})-(4.2 \mathrm{~d})$.

The efficiency of these estimates, for the particular reconstructions of Section 5 below and under the stopping criteria as evoked in Section 6 below, could be proven as in [18, 39, 49].

\section{Potential and flux reconstructions for the Robin DD in the mixed finite element method}

In this section, we propose concrete candidates for the reconstructions $\bar{s}_{h, i}^{k}, s_{h}^{k}$, and $\boldsymbol{\sigma}_{h}^{k}$ of Concepts 4.4-4.6, so that Theorem 4.7 and Corollary 4.8 become practical. Recall that $\tilde{p}_{h, i}^{k}$ is constructed from $p_{h, i}, \mathbf{u}_{h, i}$ of (3.13) by Definition 4.1.

\subsection{Potential reconstruction}

We start by $s_{h}^{k}$, which is the simplest. Let $\mathcal{T}_{\mathbf{a}}:=\left\{K \in \mathcal{T}_{h} ; \mathbf{a} \in K\right\}$ be the set of the elements $K$ that share the given vertex a from the set of vertices $\mathcal{V}_{h}$, and $\left|\mathcal{T}_{\mathbf{a}}\right|$ its cardinality. The potential reconstruction is obtained as in $[1,8,30]$ :

Definition 5.1 (Potential reconstruction). At each iteration $k$, we build the potential reconstruction $s_{h}^{k}$ by

$$
s_{h}^{k}:=\mathcal{I}_{\text {av }}\left(\tilde{p}_{h}^{k}\right),
$$

where the averaging operator $\mathcal{I}_{\text {av }}: \mathbb{P}_{2}\left(\mathcal{T}_{h}\right) \longmapsto \mathbb{P}_{2}\left(\mathcal{T}_{h}\right) \cap H^{1}(\Omega)$ associates to a piecewise 2-nd order discontinuous polynomial $\tilde{p}_{h}^{k} \in \mathbb{P}_{2}\left(\mathcal{T}_{h}\right)$ a piecewise second-order continuous polynomial $s_{h}^{k}$. The value of $s_{h}^{k} \in \mathbb{P}_{2}\left(\mathcal{T}_{h}\right) \cap H^{1}(\Omega)$ is prescribed at each Lagrange node $\mathbf{a}$ by the average of the values of $\tilde{p}_{h}^{k}$ at this node:

$$
s_{h}^{k}(\mathbf{a}):=\mathcal{I}_{\text {av }}\left(\tilde{p}_{h}^{k}\right)(\mathbf{a}):=\left.\frac{1}{\left|\mathcal{T}_{\mathbf{a}}\right|} \sum_{K \in \mathcal{T}_{\mathbf{a}}} \tilde{p}_{h}^{k}\right|_{K}(\mathbf{a}) .
$$

At the Dirichlet boundary nodes $\mathbf{a}_{\mathrm{D}} \in \Gamma^{\mathrm{D}}$, the value of $\mathcal{I}_{\mathrm{av}}\left(\tilde{p}_{h}^{k}\right)$ is set to $g_{\mathrm{D}}\left(\mathbf{a}_{\mathrm{D}}\right)$, so that $\left.s_{h}^{k}\right|_{\Gamma^{\mathrm{D}}}=g_{\mathrm{D}}$.

\subsection{Subdomain potential reconstruction}

As explained in Remarks 4.2 and 4.3, the mean values of the traces of the postprocessed mixed finite element solution $\tilde{p}_{h}^{k}$ on the edges (faces if $d=3$ ) belonging to the interface are not continuous during the DD algorithm, i.e., $\left\langle\llbracket \tilde{p}_{h}^{k} \rrbracket, 1\right\rangle_{e}$ may be different from zero for some (or even most) $e \in \mathcal{E}_{h}^{\Gamma_{i, j}}$. The purpose of this section is to construct a subdomain potential reconstruction $\bar{s}_{h, i}^{k}$ that is different from $\mathcal{I}_{\text {av }}\left(\tilde{p}_{h}^{k}\right)$ of (5.1) in that it respects this discontinuity at the beginning of the DD algorithm, but it approaches $\mathcal{I}_{\text {av }}\left(\tilde{p}_{h}^{k}\right)$ at convergence of the DD algorithm. 


\subsubsection{Notation}

We begin by introducing some more notation. The set of vertices located on the interface $\Gamma_{i, j}$ is denoted by $\mathcal{V}_{h}^{\Gamma_{i, j}} \subset \mathcal{V}_{h}$, for $i<j, \quad i, j \in \llbracket 1, \mathcal{N} \rrbracket$. Let $\mathcal{V}_{h}^{\partial \Gamma_{i, j}}$ be the set of vertices $\mathbf{a} \in \partial \Gamma_{i, j}$, and let $\mathcal{V}_{h}^{\Gamma_{i, j} \backslash\left(\partial \Gamma_{i, j}\right)}$ be the set of vertices $\mathbf{a} \in \Gamma_{i, j} \backslash\left(\partial \Gamma_{i, j}\right)$. Let $I_{\mathbf{a}}$ be the set of interfaces $\Gamma_{i, j}$ that share the vertex $\mathbf{a} \in \mathcal{V}_{h}^{\partial \Gamma_{i, j}}$ :

$$
I_{\mathbf{a}}:=\left\{\Gamma_{i, j}: i<j, \quad i, j \in \llbracket 1, \mathcal{N} \rrbracket, \mathbf{a} \in \mathcal{V}_{h}^{\partial \Gamma_{i, j}}\right\},
$$

as shown in Figure 1 for the case of a decomposition of $\Omega$ into four subdomains: $I_{\mathbf{a}}=\left\{\Gamma_{1,2}, \Gamma_{1,3}, \Gamma_{2,4}, \Gamma_{3,4}\right\}$. Let $\left|I_{\mathbf{a}}\right|$ be the cardinality of this set and let $I_{\mathbf{a}}^{r}$ be the $r$-th interface in $I_{\mathbf{a}}$ sharing $\mathbf{a}$.

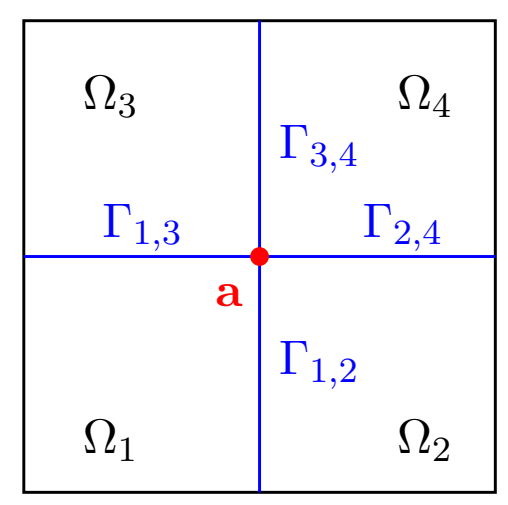

Figure 1: Intersection of the interfaces $\Gamma_{1,2}, \Gamma_{1,3}, \Gamma_{2,4}$, and $\Gamma_{3,4}$ at vertex $\mathbf{a}$

Remark that $\mathcal{T}_{\mathbf{a}}=\bigcup_{i=1}^{\mathcal{N}}\left\{K \in \mathcal{T}_{h, i} ; \mathbf{a} \in K\right\}=\bigcup_{i=1}^{\mathcal{N}} \mathcal{T}_{\mathbf{a}}^{i}$, where $\mathcal{T}_{\mathbf{a}}^{i}$ is the set of all elements in the subdomain $\Omega_{i}$ sharing the node $\mathbf{a}$; we denote by $\left|\mathcal{T}_{\mathbf{a}}^{i}\right|$ their number. We will also need $\tilde{B}^{i}$, the set of subdomains other than $\Omega_{i}$ that share at least one vertex with $\Omega_{i}$, and its cardinality $\left|\tilde{B}^{i}\right|$.

\subsubsection{Weights}

We start by defining some weights at each iteration $k$ of the DD algorithm:

Definition 5.2 (Weights of edges (faces if $d=3$ ) belonging to the interface). Define the weight of the edge (face) $e \in \mathcal{E}_{h}^{\Gamma_{i, j}}$ by

$$
\bar{w}_{e}^{k}:=\left(\frac{\left|\left\langle\llbracket \tilde{p}_{h}^{k} \rrbracket, 1\right\rangle_{e}\right|}{\left\langle\left|\llbracket \tilde{p}_{h}^{k} \rrbracket\right|, 1\right\rangle_{e}}\right)^{\alpha}, \quad \alpha \geq 1 .
$$

Note that it follows immediately from $\left|\left\langle\llbracket \tilde{p}_{h}^{k} \rrbracket, 1\right\rangle_{e}\right| \leq\left\langle\left|\llbracket \tilde{p}_{h}^{k} \rrbracket\right|, 1\right\rangle_{e}$ that

$$
0 \leq \bar{w}_{e}^{k} \leq 1 .
$$

Moreover, from what has been explained above, $\left\langle\llbracket \tilde{p}_{h}^{k} \rrbracket, 1\right\rangle_{e} \rightarrow 0$ when $k \rightarrow \infty$ on all $e \in \mathcal{E}_{h}^{\Gamma_{i, j}}$. Thus, $\bar{w}_{e}^{k}$ approaches 0 with increasing DD iterations. Conversely, $\bar{w}_{e}^{k}$ is typically close to 1 at the beginning of the DD algorithm.

Definition 5.3 (Weights of Lagrange nodes belonging to the interface). Using the notation (5.2), we define the weight on the Lagrange node $\mathbf{a} \in \mathcal{V}_{h}^{\Gamma_{i, j}}$ located on the interface (in two space dimensions for simplicity) by:

$$
\bar{w}_{\mathbf{a}}^{k}:= \begin{cases}\frac{1}{2}\left(\bar{w}_{e}^{k}+\bar{w}_{e^{\prime}}^{k}\right) & \text { if } \mathbf{a} \in \mathcal{V}_{h}^{\Gamma_{i, j} \backslash\left(\partial \Gamma_{i, j}\right)} \text { where } e, e^{\prime} \in \mathcal{E}_{h}^{\Gamma_{i, j}} / e \cap e^{\prime}=\mathbf{a}, \\ \frac{1}{\left|I_{\mathbf{a}}\right|} \sum_{r=1}^{\left|I_{\mathbf{a}}\right|} \bar{w}_{e_{r}}^{k} & \text { if } \mathbf{a} \in \mathcal{V}_{h}^{\partial \Gamma_{i, j}} \text { where } \mathbf{a} \in e_{r} \subset I_{\mathbf{a}}^{r},\end{cases}
$$

where we recall that $I_{\mathbf{a}}^{r}$ is the $r$-th interface in $I_{\mathbf{a}}$ that shares $\mathbf{a}$. 
We note that $\bar{w}_{\mathbf{a}}^{k}$ has similar properties to $\bar{w}_{e}^{k}$ : it is close to 1 at the beginning of the DD algorithm and approaches 0 during the DD iterations.

In the case of the standard averaging operator $\mathcal{I}_{\text {av }}$ from (5.1), the weights are distributed uniformly on each element $K \in \mathcal{T}_{\mathbf{a}}$ sharing the node a, being equal to $\frac{1}{\left|\mathcal{T}_{\mathbf{a}}\right|}$, see (5.1). Recall that for a given Lagrange node $\mathbf{a}$ on the interface, the patch $\mathcal{T}_{\mathbf{a}}$ is a union of subdomains subpatches $\mathcal{T}_{\mathbf{a}}^{i}$. For the subdomain potential reconstruction in the sense of Concept 4.4, we now want to define weights so that all elements sharing the same node on the interface do not have the same weight during the iterations of the DD algorithm:

Definition 5.4 (Weights of Lagrange nodes on the interface for each patch $\mathcal{T}_{\mathbf{a}}^{i}$ ). For each interface Lagrange node $\mathbf{a} \in \mathcal{V}_{h} \cap \Gamma_{i}, i \in \llbracket 1, \mathcal{N} \rrbracket$, define

$$
w_{i, \mathbf{a}}^{k}:=\frac{1}{\left|\mathcal{T}_{\mathbf{a}}^{i}\right|+\left(1-\bar{w}_{\mathbf{a}}^{k}\right) \sum_{j \in \tilde{B}^{i}}\left|\mathcal{T}_{\mathbf{a}}^{j}\right|} .
$$

The construction (5.3) ensures that at the beginning of the DD iterations, $w_{i, \mathbf{a}}^{k} \approx \frac{1}{\left|\mathcal{T}_{\mathbf{a}}^{i}\right|}$, whereas on late DD iterations, $w_{i, \mathbf{a}}^{k} \approx \frac{1}{\left|\mathcal{T}_{\mathbf{a}}\right|}$.

\subsubsection{Construction of $\bar{s}_{h, i}^{k}$}

We can now finally define:

Definition 5.5 (Subdomain potential reconstructions). At iteration $k$, for the subdomain $\Omega_{i}, i \in \llbracket 1, \mathcal{N} \rrbracket$, the subdomain potential reconstruction $\bar{s}_{h, i}^{k}$ is defined by

$$
\begin{aligned}
\bar{s}_{h, i}^{k}(\mathbf{a}) & :=\left.w_{i, \mathbf{a}}^{k} \sum_{K \in \mathcal{T}_{\mathbf{a}}^{i}} \tilde{p}_{h, i}^{k}\right|_{K}(\mathbf{a})+\left.w_{i, \mathbf{a}}^{k}\left(1-\bar{w}_{\mathbf{a}}^{k}\right) \sum_{j \in \tilde{B}^{i} K \in \mathcal{T}_{\mathbf{a}}^{j}} \sum_{h, j}\right|_{K} ^{k}(\mathbf{a}) \mathbf{a} \subset \Gamma_{i}, \\
\bar{s}_{h, i}^{k}(\mathbf{a}) & :=s_{h, i}^{k}(\mathbf{a}) \quad \text { otherwise. }
\end{aligned}
$$

Note that the sum of the weights in (5.4a) is equal to 1 for each node a. Indeed, using property (5.3),

$$
w_{i, \mathbf{a}}^{k}\left|\mathcal{T}_{\mathbf{a}}^{i}\right|+w_{i, \mathbf{a}}^{k}\left(1-\bar{w}_{\mathbf{a}}^{k}\right) \sum_{j \in \tilde{B}^{i}}\left|\mathcal{T}_{\mathbf{a}}^{j}\right|=w_{i, \mathbf{a}}^{k}\left(\left|\mathcal{T}_{\mathbf{a}}^{i}\right|+\left(1-\bar{w}_{\mathbf{a}}^{k}\right) \sum_{j \in \tilde{B}^{i}}\left|\mathcal{T}_{\mathbf{a}}^{j}\right|\right)=1 .
$$

The construction of Definition 5.5 leads to a subdomain potential reconstruction $\bar{s}_{h, i}^{k}$ where at the beginning of the DD method, the contribution of the elements of $\mathcal{T}_{\mathbf{a}}^{i}$ in the subdomain $\Omega_{i}$ is more important, with weights close to one, whereas the elements in $K \in \mathcal{T}_{\mathbf{a}} \backslash \mathcal{T}_{\mathbf{a}}^{i}$ do not contribute as their weights are close to zero. At DD convergence, all elements contribute with the same weights, so that $\bar{s}_{h, i}^{k}$ converges to $\left.\mathcal{I}_{\text {av }}\left(\tilde{p}_{h, i}^{k}\right)\right|_{\Omega_{i}}$ as the DD iterations proceed.

\subsection{Flux reconstruction}

In this section, we show how to reconstruct a flux satisfying Concept 4.6, at each iteration $k$ of the DD algorithm. We suppose that for all interface edges (faces) $e \subset \Gamma_{i, j}, \mathbf{n}_{e}$ has the same direction as the interface normal $\mathbf{n}_{\Gamma_{i, j}}$, where $\mathbf{n}_{\Gamma_{i, j}}$ is set arbitrarily, pointing either from $\Omega_{i}$ to $\Omega_{j}$, or from $\Omega_{j}$ to $\Omega_{i}$, with $j \in B^{i}$, $i<j, i \in \llbracket 1, \mathcal{N} \rrbracket$. Note first that defining simply

$$
\boldsymbol{\sigma}_{h}^{k} \cdot \mathbf{n}_{e}= \begin{cases}\left\{\mathbf{u}_{h}^{k} \cdot \mathbf{n}_{e}\right\}, & \forall e \in \underset{j \in B^{i}}{\cup} \mathcal{E}_{h}^{\Gamma_{i, j}}, \\ \mathbf{u}_{h, i}^{k} \cdot \mathbf{n}_{e}, & \forall e \in \mathcal{E}_{h, i}^{\mathcal{B n t}_{i}} \cup \mathcal{E}_{h, i}^{\text {ext }},\end{cases}
$$

we obtain the first required property (4.1a), $\boldsymbol{\sigma}_{h}^{k} \in \mathbf{H}(\operatorname{div}, \Omega)$, as well as the third property (4.1c). But the property (4.1b) does not hold in the elements having an edge (if $d=2$ ) or a face (if $d=3$ ) on the interface $\Gamma_{i, j}$. This motivates the forthcoming construction. 


\subsubsection{A simple coarse balancing problem}

Following Remarks 3.3 and 4.3 and the observation (5.5), mass balance is not preserved during the DD iterations with Robin transmission conditions. In order to restore it, a possible solution would be to use a balancing DD method like those in $[12,35,36]$, where one solves a coarse-grid problem with one unknown in each subdomain. This allows to obtain the balancing in each subdomain. We choose, however, to adopt here a new method that we find simple. It makes the connection between subdomains in order to rebalance the flux independently of the number of subdomains, and can also be applied in the case where at least one subdomain does not touch the boundary. We will more precisely define one correction per interface $\Gamma_{i, j}$ to the averaged flux $\left\{\left\{\mathbf{u}_{h}^{k} \cdot \mathbf{n}_{e}\right\}\right.$, plus some boundary corrections, through a simple coarse balancing problem. This will lead to Neumann conditions that are in equilibrium with the prescribed source term.

To explain in details our new idea, we first partition each subdomain $\Omega_{i}, \forall i \in \llbracket 1, \mathcal{N} \rrbracket$, into two disjoint parts $\Omega_{i}^{\text {ext }}$ and $\Omega_{i}^{\text {int }}$ such that $\overline{\Omega_{i}^{\text {ext }}} \cup \overline{\Omega_{i}^{\text {int }}}=\overline{\Omega_{i}}$. The so-called band $\Omega_{i}^{\text {ext }}$ is made up of simplices that have an edge, a vertex, or a face on any interface $\Gamma_{i, j}, j \in B^{i}$, see Figure 2 for a decomposition of $\Omega$ into nine subdomains. We also denote $\Gamma_{i}^{b}, b \in B^{i, \text { ext }}$, the intersections of $\partial \Omega_{i}^{\text {ext }}$ with $\partial \Omega_{i} \cap \partial \Omega$ of nonzero $(d-1)$-dimensional measure. Note that the cardinality of the index set $B^{i \text {,ext }}$ is always two in two space dimensions when $\left|\partial \Omega_{i} \cap \partial \Omega\right| \neq 0$; we let $B^{i, \text { ext }}$ empty when $\left|\partial \Omega_{i} \cap \partial \Omega\right|=0$.
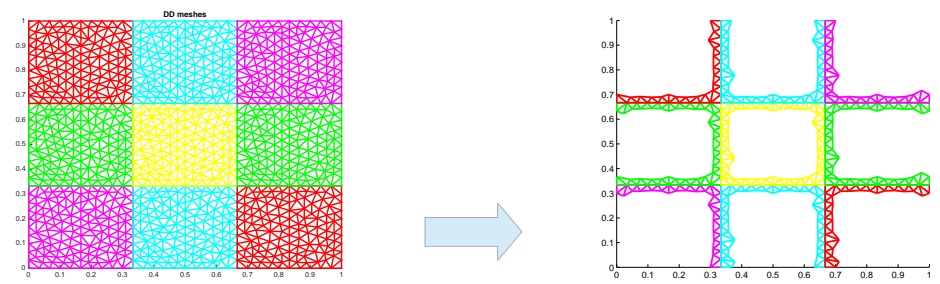

Figure 2: DD with 9 subdomains (left) and the bands $\Omega_{i}^{\text {ext }}, 1 \leq i \leq 9$ (right)

We easily see from (3.13b) that in each band $\Omega_{i}^{\text {ext }}, i \in \llbracket 1, \mathcal{N} \rrbracket$, the misfit of mass balance due to the averaging like in $(5.5)$ is

$$
\sum_{j \in B^{i}} \mathbf{n}_{\Gamma_{i, j}} \cdot \mathbf{n}_{\partial \Omega_{i}^{\text {ext }}} \sum_{e \subset \Gamma_{i, j}} \int_{e} \frac{1}{2} \llbracket \mathbf{u}_{h}^{k} \cdot \mathbf{n}_{e} \rrbracket \mathrm{d} \gamma=(f, 1)_{\Omega_{i}^{\text {ext }}}-\left\langle\left\{\left\{\mathbf{u}_{h}^{k} \cdot \mathbf{n}_{\partial \Omega_{i}^{\text {ext }}}\right\}, 1\right\rangle_{\partial \Omega_{i}^{\text {ext }}} .\right.
$$

We now try to correct the averaged interface and original boundary normal fluxes of (5.5) with one value $c_{\Gamma_{i, j}}^{k}=c_{\Gamma_{j, i}}^{k}$ per interface $\Gamma_{i, j}=\Gamma_{j, i}$ and one value $c_{\Gamma_{i}^{b}}^{k}$ per the boundary part $\Gamma_{i}^{b}$ of $\Gamma_{i}$, so that

$$
\begin{array}{ll}
c_{\Gamma_{i, j}}^{k} \approx 0 & \text { for } i, j \in \llbracket 1, \mathcal{N} \rrbracket, i<j \text { such that } j \in B^{i}, \\
c_{\Gamma_{i}^{b}}^{k} \approx 0 & \text { for } i \in \llbracket 1, \mathcal{N} \rrbracket \text { and } b \in B^{i, \text { ext }}, \text { so that }\left|\partial \Omega_{i}^{\text {ext }} \cap \partial \Omega\right|>0 .
\end{array}
$$

We keep the same value of the flux $\mathbf{u}_{h}^{k} \cdot \mathbf{n}_{\partial \Omega_{i}^{\text {ext }} \cap \partial \Omega_{i}^{\text {int }}}$ located on the boundary $\partial \Omega_{i}^{\text {ext }} \cap \partial \Omega_{i}^{\text {int }}$. We require the following $\mathcal{N}$ balancing conditions, one for each band $\Omega_{i}^{\text {ext }}$, to be satisfied:

$$
\sum_{b \in B^{i, \mathrm{ext}}} c_{\Gamma_{i}^{b}}^{k}+\sum_{j \in B^{i}}\left(\mathbf{n}_{\Gamma_{i, j}} \cdot \mathbf{n}_{\partial \Omega_{i}^{\mathrm{ext}}}\right) c_{\Gamma_{i, j}}^{k}=(f, 1)_{\Omega_{i}^{\mathrm{ext}}}-\left\langle\left\{\left\{\mathbf{u}_{h}^{k} \cdot \mathbf{n}_{\partial \Omega_{i}^{\mathrm{ext}}}\right\}, 1\right\rangle_{\partial \Omega_{i}^{\mathrm{ext}} .}\right.
$$

Equations (5.7), for $i \in \llbracket 1, \mathcal{N} \rrbracket$, lead to a rectangular linear system for the unknowns corrections $c_{\Gamma_{i, j}}^{k}$ and $c_{\Gamma_{i}^{b}}^{k}$, with more unknowns than equations. We thus look for the minimum norm solution of $(5.7)$ in the least squares sense,

$$
\sum_{i=1}^{\mathcal{N}} \sum_{b \in B^{i, \text { ext }}}\left(c_{\Gamma_{i}^{b}}^{k}\right)^{2}+\sum_{i=1}^{\mathcal{N}} \sum_{j \in B^{i}, i<j}\left(c_{\Gamma_{i, j}}^{k}\right)^{2}=\min
$$

In the example of Figure 2, there are 12 corrections $c_{\Gamma_{i, j}}^{k}$ according to (5.6a) and 16 corrections $c_{\Gamma_{i}^{b}}^{k}$ according to $(5.6 \mathrm{~b})$ to be found. As there are 9 subdomains, there are 28 unknowns and 9 equations (5.7) to be satisfied here, in the least-squares sense (5.8). 
Conditions (5.7) immediately give mass balance in each band $\Omega_{i}^{\text {ext }}$ (and consequently in each subdomain $\left.\Omega_{i}\right)$

Lemma 5.6. Adding the corrections $c_{\Gamma_{i, j}}^{k}$ and $c_{\Gamma_{i}^{b}}^{k}$ of (5.6) constructed via conditions (5.7)-(5.8) to the averaged fluxes $\left\{\mathbf{u}_{h}^{k} \cdot \mathbf{n}_{\Omega_{i}^{\text {ext }}}\right\}$ leads to mass balance in each band $\Omega_{i}^{\text {ext }}, \forall i \in \llbracket 1, \mathcal{N} \rrbracket$ :

$$
\begin{aligned}
& \sum_{b \in B^{i, \text { ext }}}\left(\left\langle\mathbf{u}_{h}^{k} \cdot \mathbf{n}_{\Omega_{i}^{\text {ext }}}, 1\right\rangle_{\Gamma_{i}^{b}}+c_{\Gamma_{i}^{b}}^{k}\right)+\left\langle\left\{\left\{\mathbf{u}_{h}^{k} \cdot \mathbf{n}_{\Omega_{i}^{\text {ext }}}\right\}, 1\right\rangle_{\partial \Omega_{i}^{\text {ext }} \cap \partial \Omega_{i}^{\text {int }}}\right. \\
& +\sum_{j \in B^{i}}\left(\left\langle\left\{\left\{\mathbf{u}_{h}^{k} \cdot \mathbf{n}_{\Omega_{i}^{\text {ext }}}\right\}\right\}, 1\right\rangle_{\Gamma_{i, j}}+\left(\mathbf{n}_{\Gamma_{i, j}} \cdot \mathbf{n}_{\partial \Omega_{i}^{\text {ext }}}\right) c_{\Gamma_{i, j}}^{k}\right)=(f, 1)_{\Omega_{i}^{\text {ext }}} .
\end{aligned}
$$

\subsubsection{Solving local Neumann problems in bands around the interfaces}

Building on the key balancing property (5.9), we can now follow [39, Section 3.5.2] and solve a well-posed local Neumann problem in each band $\Omega_{i}^{\text {ext }}$. This will lead to a flux reconstruction in the sense of Concept 4.6. The procedure is as follows:

Definition 5.7 (Spaces of local Neumann problem). For $i \in \llbracket 1, \mathcal{N} \rrbracket$, define $M_{h, i}\left(\Omega_{i}^{\text {ext }}\right)$ as the restriction of $M_{h, i}$ to $\Omega_{i}^{\text {ext }}$ and

$$
\begin{aligned}
& \mathbf{W}_{h, z, \Omega_{i}^{\text {ext }}}=\left\{\mathbf{v}_{h}^{k} \in \mathbf{W}_{h, i}\left(\Omega_{i}^{\text {ext }}\right) ;\right. \\
& \mathbf{v}_{h}^{k} \cdot \mathbf{n}_{\Omega_{i}^{\text {ext }}}=z+\mathbf{n}_{\partial \Omega_{i}^{\text {ext }}} \cdot \mathbf{n}_{\Gamma_{i, j}} \frac{c_{\Gamma_{i, j}}^{k}\left|\Gamma_{i, j}\right|}{\Gamma_{i}} \text { if } z \neq \star, 0 \text { else }, \quad \text { on } \Gamma_{i, j}, j \in B^{i}, \\
& \mathbf{v}_{h}^{k} \cdot \mathbf{n}_{\Omega_{i}^{\text {ext }}}=z+\frac{c_{\Gamma_{i}^{b}}^{k}}{\left|\Gamma_{i}^{b}\right|} \text { if } z \neq \star, 0 \text { else, } \quad \text { on } \Gamma_{i}^{b}, b \in B^{i, \text { ext }}, \\
& \left.\mathbf{v}_{h}^{k} \cdot \mathbf{n}_{\Omega_{i}^{\text {ext }}}=z \text { if } z \neq \star, 0 \text { else } \quad \text { on } \partial \Omega_{i}^{\text {ext }} \cap \partial \Omega_{i}^{\text {int }}\right\} .
\end{aligned}
$$

Definition 5.8 (Mixed finite element local Neumann problem in the bands). For all $i \in \llbracket 1, \mathcal{N} \rrbracket$, find

$$
\left.\boldsymbol{\sigma}_{h}^{k}\right|_{\Omega_{i}^{\text {ext }}} \in \mathbf{W}_{h,\left\{\mathbf{u}_{h}^{k} \cdot \mathbf{n}_{\Omega_{i}^{\text {ext }}}\right\}, \Omega_{i}^{\text {ext }}} \text { and } q_{h}^{k} \in M_{h, i}\left(\Omega_{i}^{\text {ext }}\right) \text {, with }\left.\left(q_{h}^{k}, 1\right)\right|_{\Omega_{i}^{\text {ext }}}=0,
$$

such that

$$
\begin{aligned}
& \left(\boldsymbol{S}^{-1}\left(\boldsymbol{\sigma}_{h}^{k}-\mathbf{u}_{h}^{k}\right), \mathbf{v}_{h}\right)_{\Omega_{i}^{\text {ext }}}-\left(q_{h}^{k}, \nabla \cdot \mathbf{v}_{h}\right)_{\Omega_{i}^{\text {ext }}}=0, \quad \forall \mathbf{v}_{h} \in \mathbf{W}_{h, \star, \Omega_{i}^{\text {ext }}}, \\
& \left(\nabla \cdot \boldsymbol{\sigma}_{h}^{k}, w_{h}\right)_{\Omega_{i}^{\text {ext }}}=\left(f, w_{h}\right)_{\Omega_{i}^{\text {ext }}}, \quad \forall w_{h} \in M_{h, i}\left(\Omega_{i}^{\text {ext }}\right) \text { with }\left(w_{h}, 1\right)_{\Omega_{i}^{\text {ext }}}=0 .
\end{aligned}
$$

\subsubsection{Construction of $\sigma_{h}^{k}$}

We finally set, for all $i \in \llbracket 1, \mathcal{N} \rrbracket$,

$$
\boldsymbol{\sigma}_{h}^{k}:=\left\{\begin{array}{ll}
\left.\boldsymbol{\sigma}_{h}^{k}\right|_{\Omega_{i}^{\text {ext }}} & \text { on } \Omega_{i}^{\text {ext }} \text { by Definition } 5.8, \\
\left.\mathbf{u}_{h}^{k}\right|_{\Omega_{i}^{\text {int }}} & \text { on } \Omega_{i}^{\text {int }} .
\end{array} \text { for all } i \in \llbracket 1, \mathcal{N} \rrbracket .\right.
$$

This is our flux reconstruction $\boldsymbol{\sigma}_{h}^{k}$ satisfying all conditions of Concept 4.6.

\section{$6 \quad$ Numerical results}

In this section, we give some numerical illustrations of the a posteriori error estimators of Theorem 4.7 and Corollary 4.8 , in two space dimensions. 


\subsection{A homogeneous, anisotropic medium}

We set $\Omega=] 0,1[\times] 0,1\left[\right.$ and consider $x=0$ as the Neumann boundary $\Gamma^{\mathrm{N}}, y=0$ and $y=1$ as the Dirichlet boundaries $\Gamma^{\mathrm{D}}$, and $x=1$ as the Robin boundary, in extension of (1.1), together with the diffusion tensor $\boldsymbol{S}=\left(\begin{array}{ll}3 & 2 \\ 2 & 3\end{array}\right)$. We choose the right-hand side $f$ and the values of the boundary conditions so that the exact solution is given by $p(x, y)=\sin (2 \pi x) \sin (2 \pi y)$. We consider $\Omega$ divided into 9 regular subdomains, as in Figure 2, left. The number of triangles in the whole domain $\Omega$ is 115200 . The Robin parameters of the DD algorithm are optimized following [22]. We consider the mixed finite element discretization (3.13) in two cases:

\subsubsection{Solution with block-Jacobi}

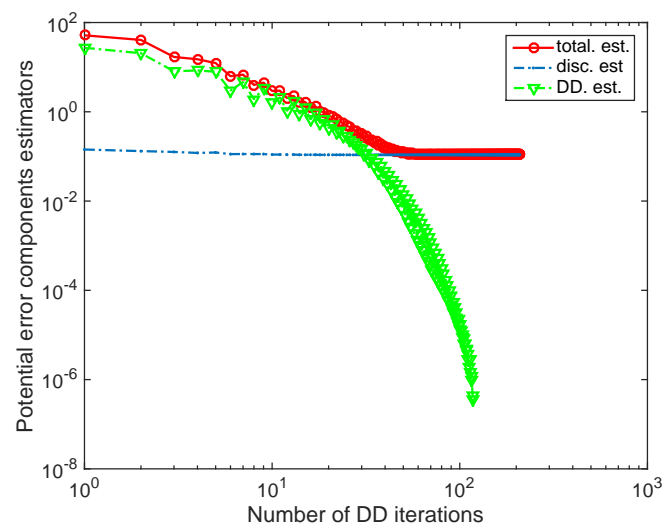

Figure 3: Example 1: error component estimates with the block-Jacobi solver

We first consider the block-Jacobi DD solver of Section 3.2. In Figure 3, we plot the evolution of the estimators $\eta_{\mathrm{DD}}^{k}$ and $\eta_{\mathrm{disc}, p}^{k}$ of Corollary 4.8 and of their sum $\tilde{\eta}^{k}$ as a function of the number of the block-Jacobi DD iterations. The original DD stopping criterion is when the jump of the Robin condition measured in the $L^{2}$ norm on the interface has been reduced below $10^{-12}$, which is satisfied after 209 iterations. At the beginning we see that $\eta_{\mathrm{DD}}^{k}$ dominates up to roughly 35 iterations and then gets smaller compared to $\eta_{\mathrm{disc}, p}^{k}$ and then vanishes. The stopping criterion for the iterative solver that we propose instead is to stop when the domain decomposition error does not contribute significantly to the overall error, i.e., $\eta_{\mathrm{DD}}^{k} \leq \gamma \eta_{\text {disc }, p}^{k}$, with $\gamma \approx 0.1$. Here, we can stop at iteration 47, and avoid 162 unnecessary iterations. Thus, we can spare $77.5 \%$ of the total number of iterations.
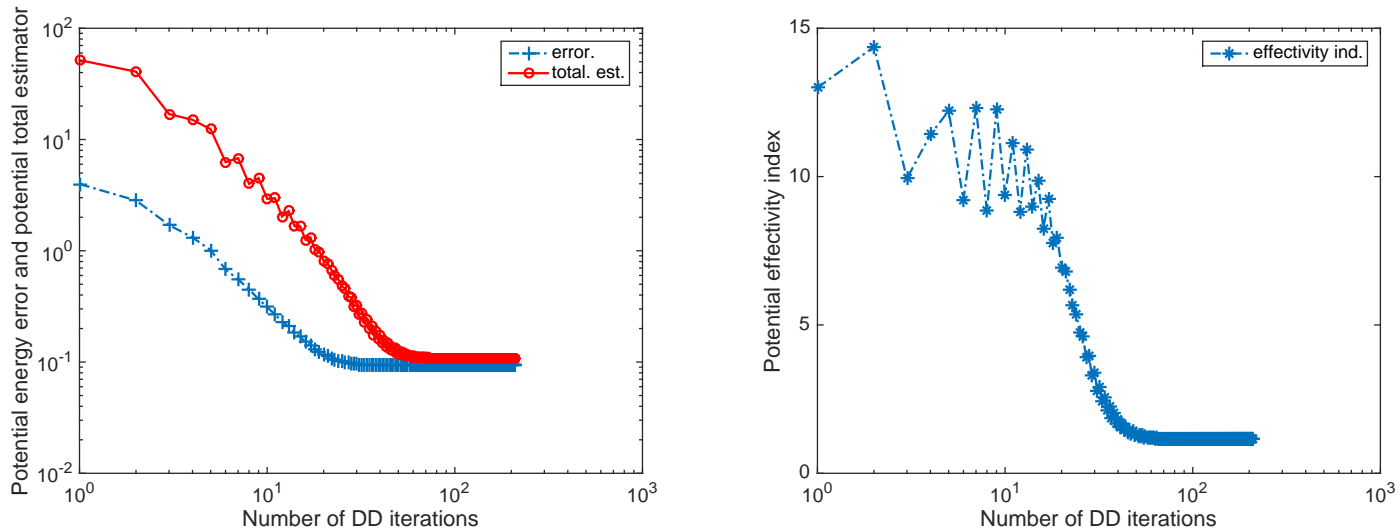

Figure 4: Example 1: energy error and total estimator (left) and the effectivity index (right) with the block-Jacobi solver 
We also plot the energy error and the total estimator as a function of the number of iterations, see Figure 4 on the left. Consequently, we can obtain the effectivity index

$$
I_{\mathrm{eff}}^{k}:=\frac{\tilde{\eta}^{k}}{\left\|p-\tilde{p}_{h}^{k}\right\| \mid}
$$

from Corollary 4.8 defined as the ratio of the estimated and the actual error at the iteration $k$ of the DD algorithm, see Figure 4 on the right. We observe that the effectivity index approaches the optimal value of 1.

\section{Discussion of the estimates at iteration 47}

At iteration 47 , the solution $p_{h}^{47}$ does not present any visual discontinuity, see Figure 5. Figure 6 shows that the element contributions of $\eta_{\text {disc }, p}^{k}$ are about the size of $5.10^{-4}$ and are distributed rather uniformly over the whole domain, while $\eta_{\mathrm{DD}}^{k}$ is about $10^{-3}$ and is distributed only around the interfaces. We can see in Figure 7 (left) that the total error estimator distribution is very close to the distribution of the estimator $\eta_{\text {disc, }, p}^{k}$, up to the error on the interface. Finally, we see that the energy error distribution shown in Figure 7 (right) matches well with the total error estimator distribution, see Figure 7 (left) (again, up to the error on the interface).

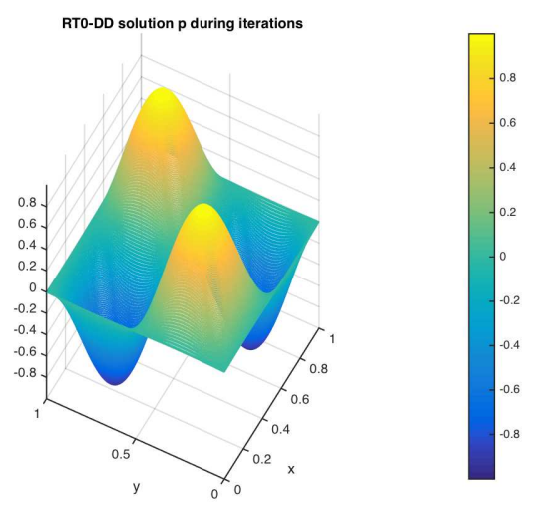

Figure 5: Example 1: pressure at the 47th iteration with the block-Jacobi solver
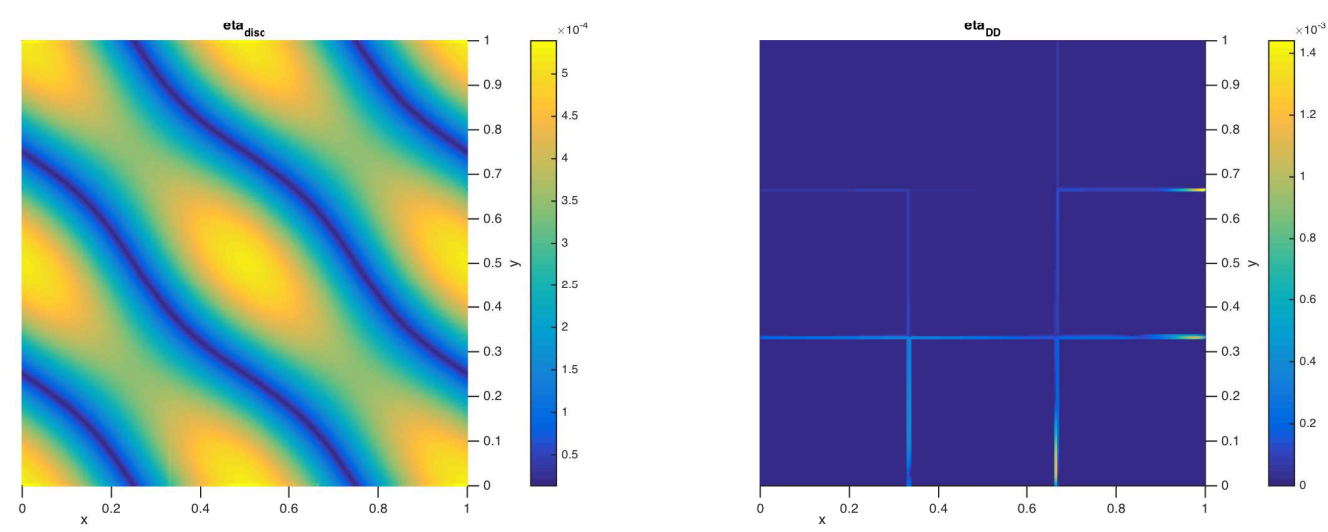

Figure 6: Example 1: the two components of the a posteriori estimates $\eta_{\mathrm{disc}, p}^{k}$ (left) and $\eta_{\mathrm{DD}}^{k}$ (right) on each element $K$ of $\mathcal{T}_{h}$, at the 47 th iteration with the block-Jacobi solver 

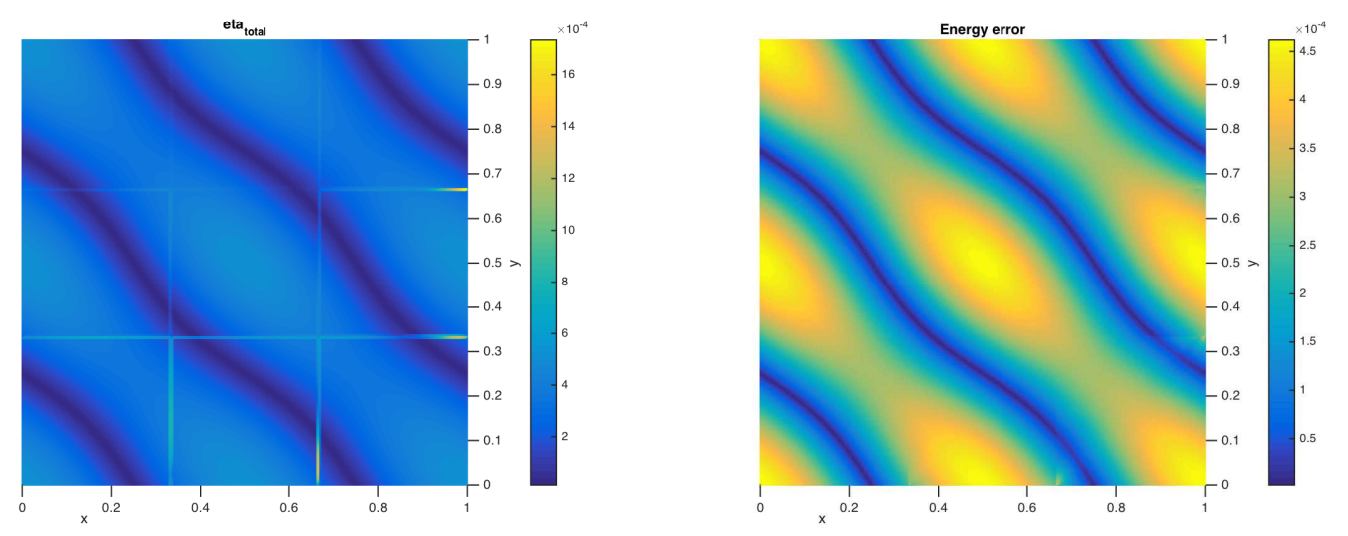

Figure 7: Example 1: the total error estimator (left) and the distribution of the energy error (right) at the 47th iteration with the block-Jacobi solver

\subsubsection{Solution with GMRES}

We take the same example as before but using the GMRES solver of Section 3.3 now. One particular advantage of the GMRES solver is that it typically takes fewer iterations than the block-Jacobi for the same original DD stopping criteria (when the residual given by the jump of the Robin condition is lower than $10^{-12}$ on the interface), and which is verified here after 61 iterations. As shown in Figure 8 (left), $\eta_{\mathrm{DD}}^{k}$ dominates up to roughly 12 iterations and then gets small compared to $\eta_{\text {disc }, p}^{k}$. Using the stopping criterion $\eta_{\mathrm{DD}}^{k} \leq 0.1 \eta_{\mathrm{disc}, p}^{k}$, we can stop the DD algorithm at iteration 17 , and thus save 44 unnecessary iterations. Thus, we can spare $72 \%$ of the total number of iterations. We finally plot the energy error and the total estimator as a function of the number of iterations, see Figure 8 (right). Consequently, we can obtain the effectivity index $I_{\mathrm{eff}}^{k}$ at each iteration of the DD algorithm, which is again close to the optimal value of 1.
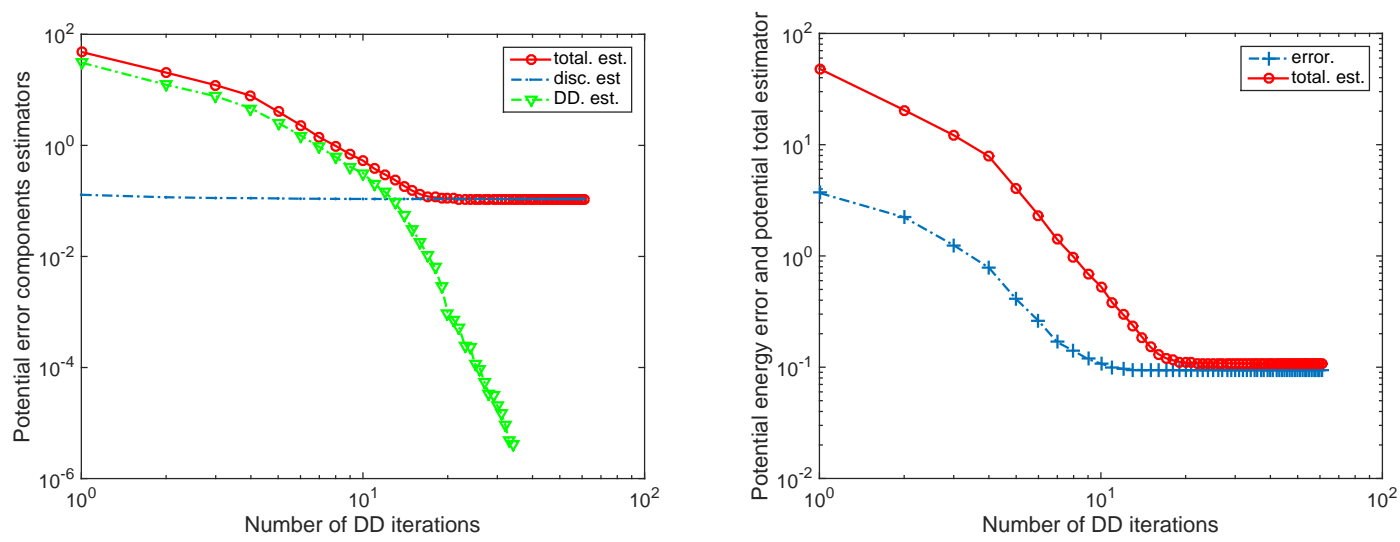

Figure 8: Example 1: error component estimates (left) and energy error and total estimator (right) with the GMRES solver

\subsection{A heterogeneous, isotropic medium}

The second example focuses on the approximation of problem $(1.1)$ where $\Omega=] 0,1[\times] 0,1[, \partial \Omega$ is the Dirichlet boundary, $p(x, y)=x(1-x) y(1-y)$ is the exact solution, and where the diffusion tensor is

$$
\boldsymbol{S}= \begin{cases}15-10 \sin (10 \pi x) \sin (10 \pi y) \boldsymbol{I}, & x, y \in(0,1 / 2) \text { or } x, y \in(1 / 2,1), \\ 15-10 \sin (2 \pi x) \sin (2 \pi y) \boldsymbol{I}, & \text { otherwise, }\end{cases}
$$


where $\boldsymbol{I}$ is the ( 2 by 2 ) identity matrix. We consider a domain decomposition of $\Omega$ into 4 subdomains $\Omega=\cup_{i=1}^{4} \Omega_{i}$. The interface problem is solved with GMRES of Section 3.3.

In this example, we can see from Figure 9 that we can stop after 6 iterations, and so save 34 unnecessary iterations. We also plot the energy error and the total estimator as a function of the number of iterations, see Figure 10. We again observe that the effectivity index is close to the optimal value of 1 .

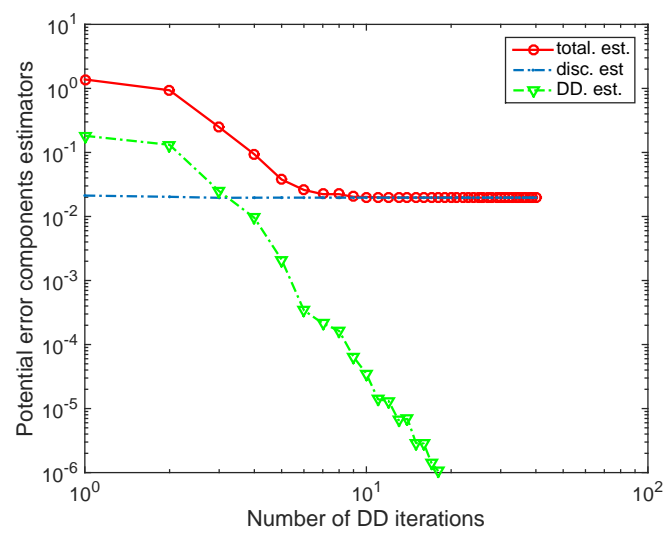

Figure 9: Example 2: error component estimates with the GMRES solver
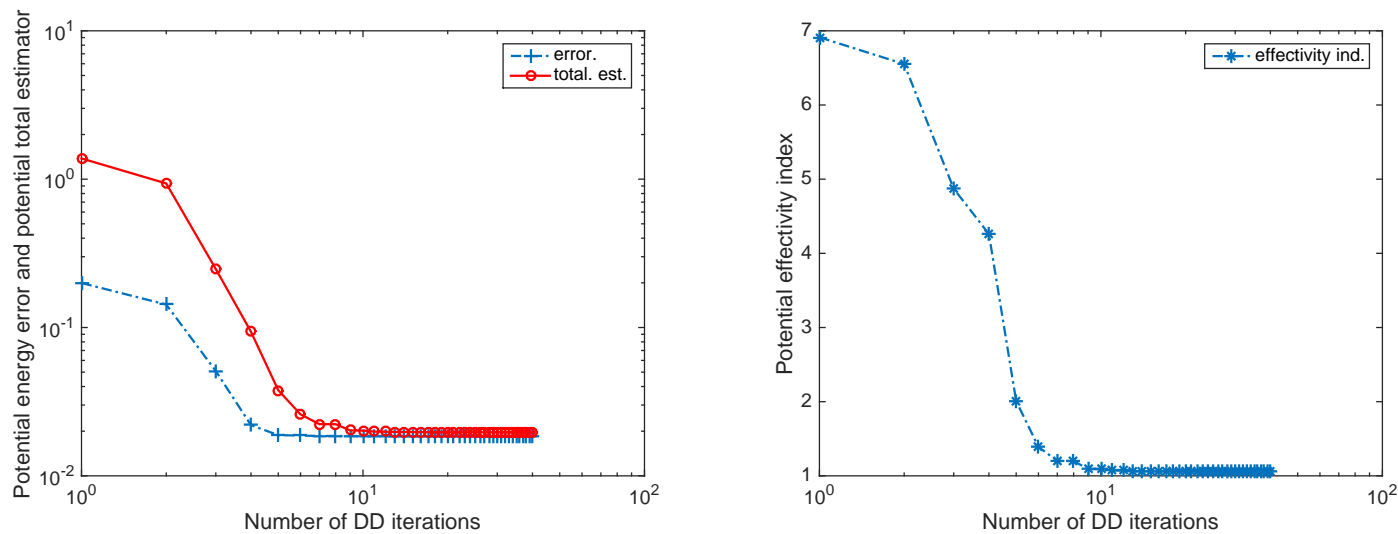

Figure 10: Example 2: energy error and total estimator (left) and the effectivity index (right) with the GMRES solver

\section{Discussion of the estimators at iteration 6}

At iteration 6, we remark that the DD error is located on the interface, see Figure 11 (top right). We can see in Figure 11 (bottom left) that the total error estimator distribution is very close to the error distribution of $\eta_{\text {disc }, p}^{k}$ in Figure 11 (top left). Finally, we see that the energy error distribution in Figure 11 (bottom right) matches well with the total error estimator distribution, see Figure 11 (bottom left). 

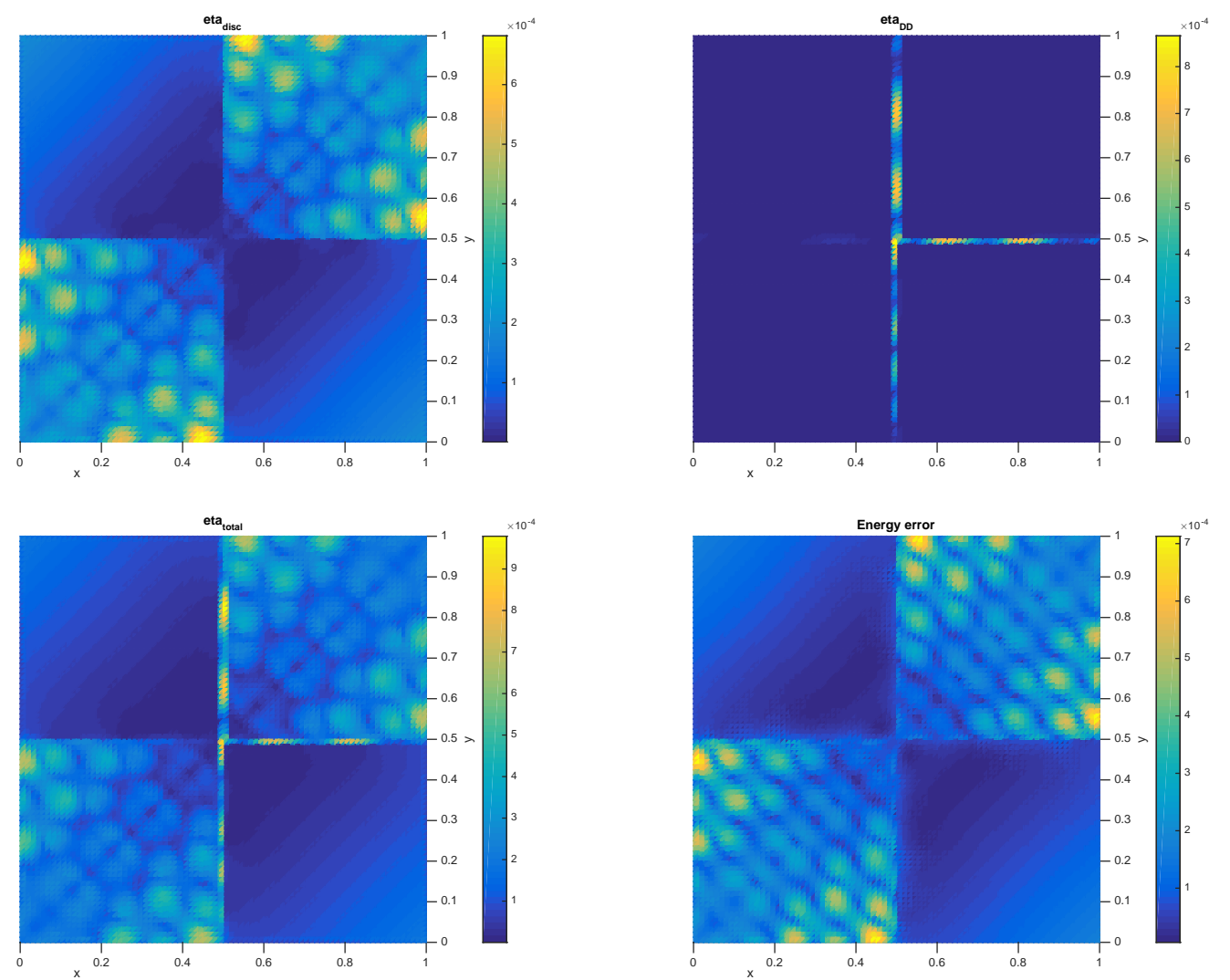

Figure 11: Example 2: the two components of the a posteriori estimates $\eta_{\text {disc }, p}^{k}$ (top left) and $\eta_{\mathrm{DD}}^{k}$ (top right) on each element $K$ of $\Omega$, the total error estimator (bottom left) and the distribution of the energy error (bottom right), at the 6th iteration of the GMRES solver

\subsection{Singular solution: adaptive DD and mesh refinement}

In this last subsection, we combine the adaptive stopping criteria with adaptive mesh refinement. We are in particular interested in the interplay of the domain decomposition and discretization error components in such a combined procedure.

We consider the L-shaped domain $\Omega=(-1,1) \times(-1,1) \backslash[0,1] \times[-1,0]$ with $\partial \Omega$ the Dirichlet boundary, $f=$ $0, \boldsymbol{S}=\boldsymbol{I}$, and the exact solution (in polar coordinates) $p(r, \varphi)=r^{\frac{2}{3}} \sin \left(\frac{2 \varphi}{3}\right)$. The domain is decomposed into three subdomains: $\bar{\Omega}=\cup_{i=1}^{3} \bar{\Omega}_{i}$, where $\Omega_{1}=(-1,0) \times\left(-1,-\frac{1}{2}\right)$ (blue), $\Omega_{2}=\left(-1, \frac{1}{2}\right) \times\left(-\frac{1}{2}, 1\right) \backslash\left[0, \frac{1}{2}\right] \times\left[-\frac{1}{2}, 0\right]$ (cyan), and $\Omega_{3}=\left(\frac{1}{2}, 1\right) \times(0,1)$ (yellow), as shown in Figure 12 .

In what follows, we will consider a sequence of grids $\left\{\mathcal{T}_{h}^{(\ell)}\right\}_{\ell \geq 0}$, and denote by $\mathcal{E}_{h}^{(\ell), \Gamma_{i, j}}$ the set of edges (faces) of $\mathcal{T}_{h}^{(\ell)}$ on $\Gamma_{i, j}$. For two grids $\mathcal{T}_{h}^{(\ell)}$ and $\mathcal{T}_{h}^{(\ell+1)}$ we will also introduce $\Pi_{\ell+1, \ell}$ such that $\left.\Pi_{\ell+1, \ell}\right|_{\Gamma_{i, j}}$ is the $L^{2}$ projection from piecewise constant functions on $\mathcal{E}_{h}^{(\ell), \Gamma_{i, j}}$ onto piecewise constant functions on $\mathcal{E}_{h}^{(\ell+1), \Gamma_{i, j}}$. Then, the DD algorithm with adaptive stopping criteria and adaptive mesh refinement is, for a given tolerance $\delta$, defined as follows:

We set $\delta=6.10^{-3}$ and start Algorithm 1 with an initial grid $\mathcal{T}_{h}^{(0)}$ shown in Figure 12 (on the left), and with an initial guess $\boldsymbol{\xi}_{h}^{(0)}$ equal to zero on $\Gamma$. Then Algorithm 1 stops after 2 iterations. Figure 12 shows the resulting adapted meshes: $\mathcal{T}_{h}^{(1)}$ (in the middle) and $\mathcal{T}_{h}^{(2)}$ (on the right).

Table 1 summarizes the discretization data, recalls the stopping criterion for the DD solver, and presents the effectivity indices on the last DD iteration and the relative $L^{2}$ pressure and flux errors for the three 
Data: Enter the initial grid $\mathcal{T}_{h}^{(0)}$ and a Robin initial guess $\boldsymbol{\xi}_{h}^{(0)}$ on $\Gamma$

Result: The pressure head $p_{h}^{(\ell)}$ and flux $\mathbf{u}_{h}^{(\ell)}$ on $\mathcal{T}_{h}^{(\ell)}$

$\ell:=0$; repeat Mesh refinement iterations

$k:=0$; Initialize GMRES with $\boldsymbol{\xi}_{h}^{\ell, 0}=\boldsymbol{\xi}_{h}^{(\ell)}$ on $\Gamma$ :

repeat GMRES DD iterations

Perform one GMRES iteration on the discrete counterpart of Problem (3.9) on $\mathcal{T}_{h}^{(\ell)}$;

Compute $\eta_{\mathrm{DD}}^{k}, \eta_{\mathrm{disc}, p}^{k}$, and $\tilde{\eta}^{k}$ from Corollary 4.8 at iteration $k$;

Set $\eta_{\mathrm{DD}}^{\ell, k}:=\eta_{\mathrm{DD}}^{k}, \eta_{\mathrm{disc}, p}^{\ell, k}:=\eta_{\mathrm{disc}, p}^{k}$, and $\tilde{\eta}^{\ell, k}:=\tilde{\eta}^{k}$

$k \longleftarrow k+1$;

until $\eta_{\mathrm{DD}}^{\ell, k} \leq 0.1 \eta_{\mathrm{disc}, p}^{\ell, k}$

$\triangleright$ Denote by $K_{\ell}$ the last GMRES iteration, $\boldsymbol{\xi}_{h}^{(\ell)}$ the discrete Robin solution on $\Gamma$ at iteration $K_{\ell}$, and set $\eta_{\mathrm{DD}}^{(\ell)}:=\eta_{\mathrm{DD}}^{\ell, K_{\ell}}, \eta_{\mathrm{disc}, p}^{(\ell)}:=\eta_{\mathrm{disc}, p}^{\ell, K_{\ell}}$, and $\tilde{\eta}^{\ell}:=\tilde{\eta}^{\ell, K_{\ell}}$;

$\triangleright$ Construct a new grid $\mathcal{T}_{h}^{(\ell+1)}$ from $\mathcal{T}_{h}^{(\ell)}$ and the indicators $\eta_{\text {disc }, p}^{(\ell)}{ }^{1}$;

$\triangleright$ Compute a new Robin initial guess $\boldsymbol{\xi}_{h}^{(\ell+1)}=\Pi_{\ell+1, \ell}\left(\boldsymbol{\xi}_{h}^{(\ell)}\right)$;

Compute $\left(p_{h}^{(\ell)}, \mathbf{u}_{h}^{(\ell)}\right)$ from $\boldsymbol{\xi}_{h}^{(\ell)}$ by solving the discrete couterpart of problem (3.4) in each subdomain, with $\xi_{i, j}:=\left.\boldsymbol{\xi}_{h}^{(\ell)}\right|_{\Gamma_{i, j}}$.

$\ell \longleftarrow \ell+1$

until $\tilde{\eta}^{\ell} \leq \delta$

Algorithm 1: Adaptive DD algorithm with mesh refinement
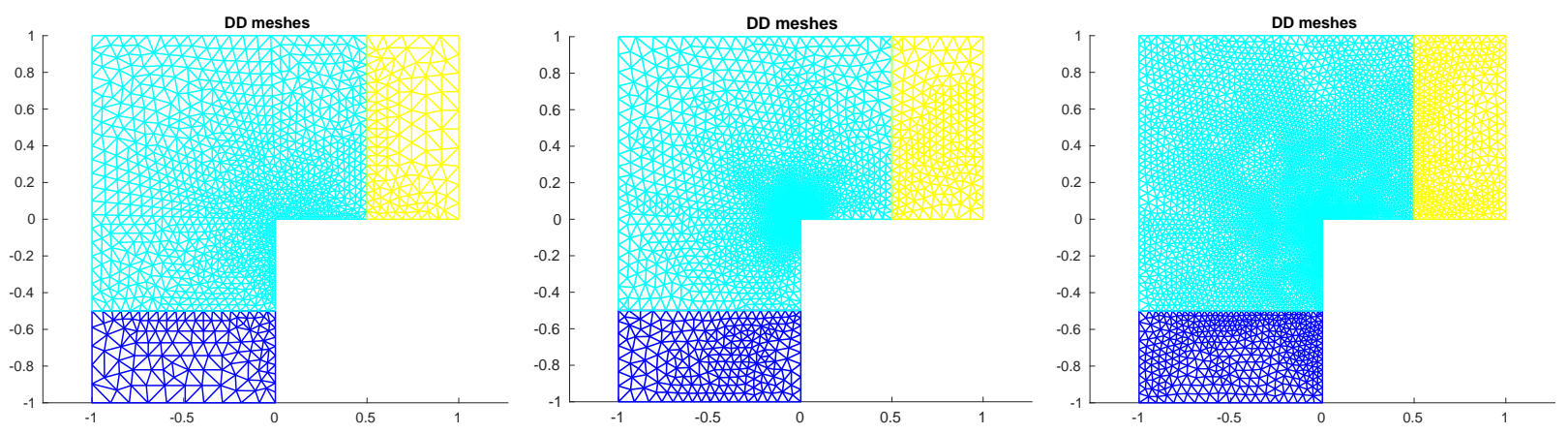

Figure 12: Example 3: Adapted meshes of $\Omega$ : $\mathcal{T}_{h}^{(0)}$ (left), $\mathcal{T}_{h}^{(1)}$ (middle) and $\mathcal{T}_{h}^{(2)}$ (right), and domain decomposition: $\Omega_{1}$ (blue), $\Omega_{2}$ (cyan), and $\Omega_{3}$ (yellow)

meshes $\mathcal{T}_{h}^{(\ell)}, \ell=0,1,2$.

\begin{tabular}{lrrr}
\hline & $\ell=0$ & $\ell=1$ & $\ell=2$ \\
\hline Number of triangles in $\Omega_{1}$ & 227 & 390 & 782 \\
Number of triangles in $\Omega_{2}$ & 2170 & 4020 & 8581 \\
Number of triangles in $\Omega_{3}$ & 227 & 391 & 804 \\
A posteriori stopping criterion & $\eta_{\mathrm{DD}}^{(0)} \leq 0.1 \eta_{\text {disc, }, p}^{(0)}$ & $\eta_{\mathrm{DD}}^{(1)} \leq 0.1 \eta_{\text {disc }, p}^{(1)}$ & $\eta_{\mathrm{DD}}^{(2)} \leq 0.1 \eta_{\text {disc }, p}^{(2)}$ \\
Number of GMRES iterations & 7 & 4 & 5 \\
Effectivity index & 1.3446 & 1.2401 & 1.1880 \\
rel. $\left\|p-p_{h}^{(\ell)}\right\|_{L^{2}(\Omega)}$ & 0.01726 & 0.014371 & 0.0090242 \\
rel. $\left\|\mathbf{u}-\mathbf{u}_{h}^{(\ell)}\right\|_{L^{2}(\Omega)}$ & 0.035368 & 0.0212 & 0.013142 \\
\hline
\end{tabular}

Table 1: Example with adaptive stopping criteria and three mesh refinements

Figure 13 shows the evolution of the estimators $\eta_{\mathrm{DD}}^{\ell, k}$ and $\eta_{\mathrm{disc}, p}^{\ell, k}$ and of their sum $\tilde{\eta}^{\ell, k}$ as a function of the number of GMRES iterations $k$, for $\ell=0$ (left), $\ell=1$ (middle), and $\ell=2$ (right). We observe that $\tilde{\eta}^{\ell, 1}$ is 
improved (note the scale changes) as well as the number of DD iterations, as the global grid is adaptively refined and the discretization error is reduced.
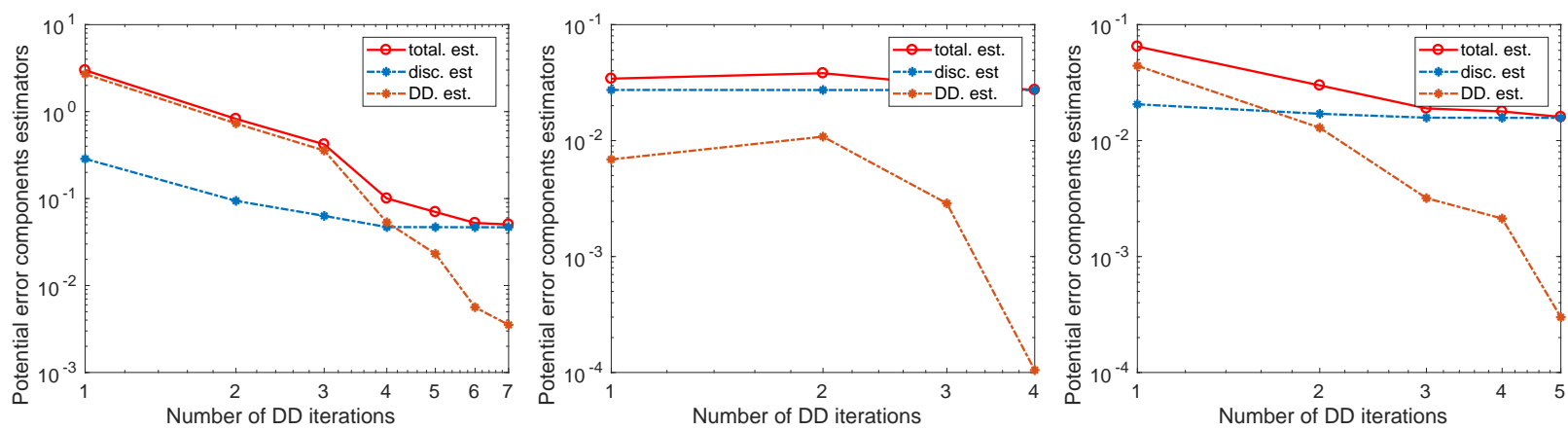

Figure 13: Example 3: error component estimates for different refinements: $\ell=0$ (left), $\ell=1$ (middle), and $\ell=2$ (right). Note that the scale changes from one figure to the next.

Figure 14 (top) shows the energy error and the total estimator as a function of the number of iterations, for $\ell=0$ (left), $\ell=1$ (middle), and $\ell=2$ (right). We observe that the total estimator becomes closer to the energy error, especially on the first DD iterations, as the global grid is adaptively refined and the discretization error is reduced. Thus, we can obtain the effectivity index

$$
I_{\mathrm{eff}}^{\ell, k}:=\frac{\tilde{\eta}^{\ell, k}}{\left\|p-\tilde{p}_{h}^{\ell, k}\right\| \mid}
$$

(with $\tilde{p}_{h}^{\ell, k}$ the postprocessing of $p_{h}^{\ell, k}$ ), as a function of the number of GMRES iterations $k$, for $\ell=0$ (left), $\ell=1$ (middle), and $\ell=2$ (right), see Figure 14 (bottom). We observe that the effectivity index better approaches the optimal value of 1 , as the global grid is adaptively refined and the discretization error is reduced.
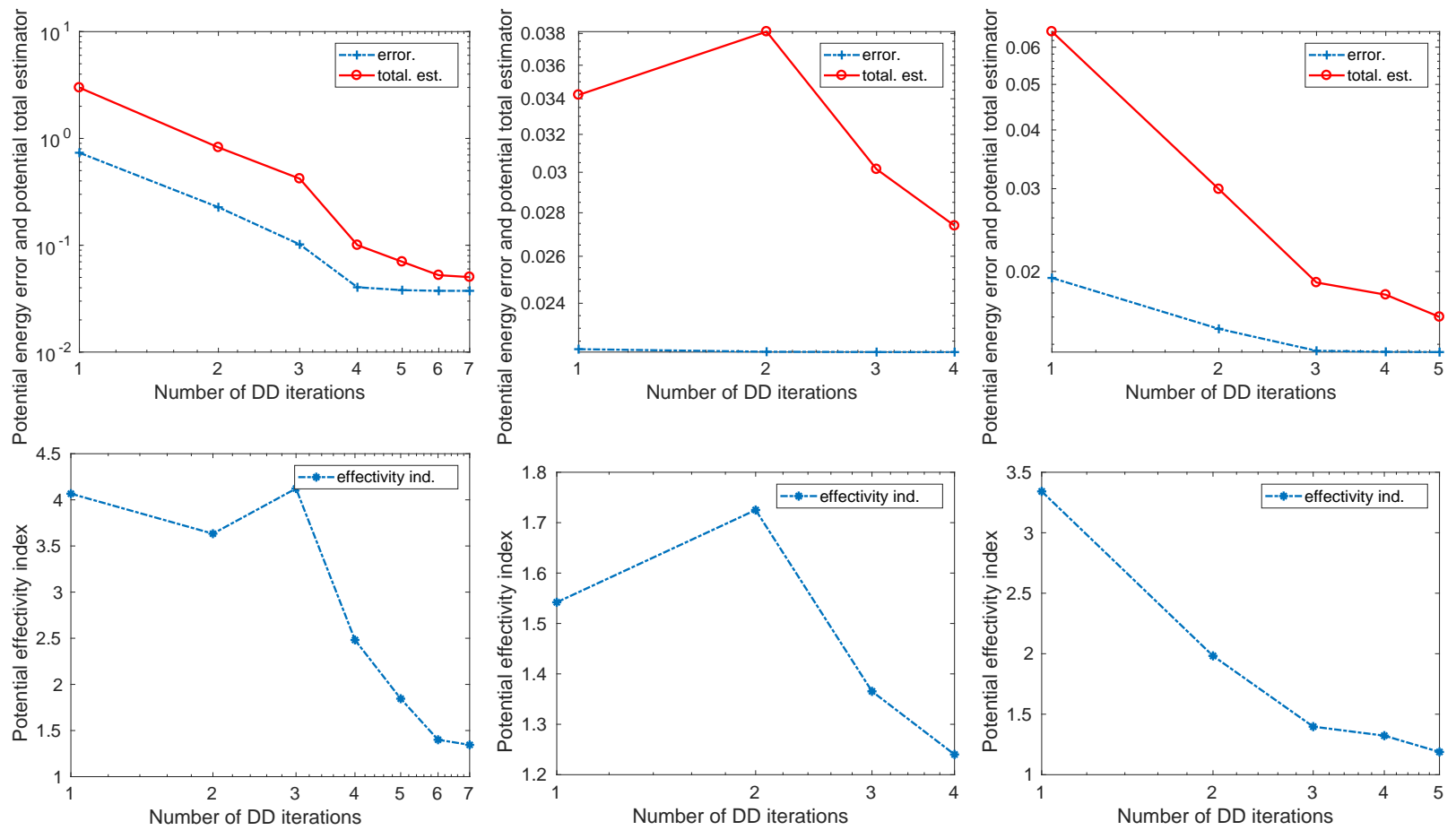

Figure 14: Example 3: energy error and total estimator (top) and effectivity index (bottom), for different refinements: $\ell=0$ (left), $\ell=1$ (middle), and $\ell=2$ (right). 
Figure 15 shows the element contributions of $\eta_{\mathrm{DD}}^{\ell, K_{\ell}}$ (top) and of $\eta_{\text {disc, }, p}^{\ell, K_{\ell}}$ (bottom), at the last GMRES iteration $K_{\ell}$, for $\ell=0$ (left), $\ell=1$ (middle), and $\ell=2$ (right). The former are distributed only around the interfaces, while the latter are distributed only around the corner $(0,0)$, for all refinements $\ell=0,1,2$.
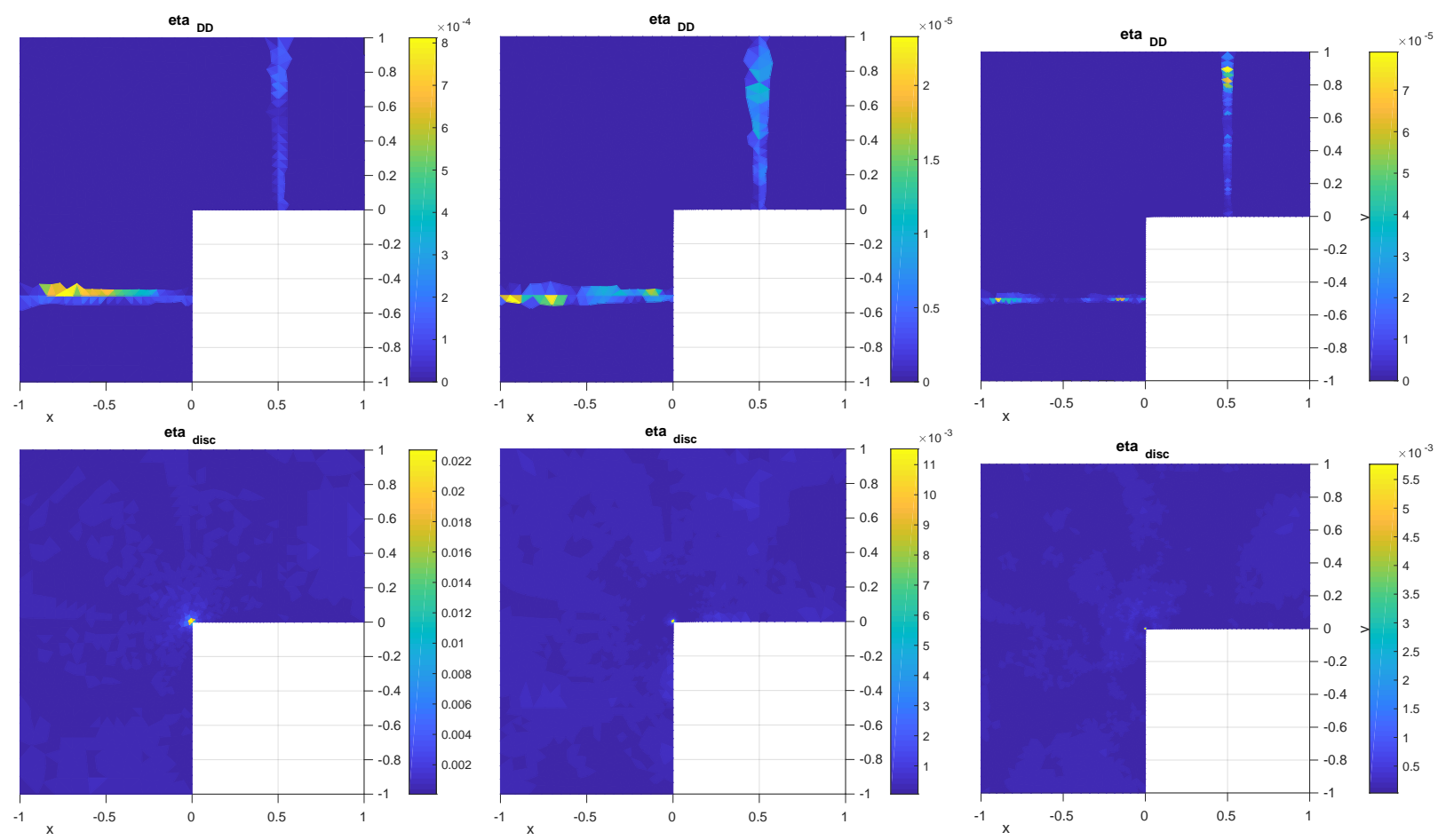

Figure 15: Example 3: the components $\eta_{\mathrm{DD}}^{\ell, k}$ (top) and $\eta_{\text {disc, }, ~}^{\ell, k}$ (bottom) of the a posteriori estimates on each element $K$ of $\Omega$, for different refinements: $\ell=0$ (left), $\ell=1$ (middle), and $\ell=2$ (right).

We can see in Figure 16 (top) that at each refinement $\ell=0,1,2$, the total error estimator distribution is very close to the distribution of the discretization estimator, up to the error on the interface. Finally, we see that the energy error distribution shown in Figure 16 (bottom) matches well with the total error estimator distribution of Figure 16 (top) (again, up to the error on the interface), at each refinement $\ell=0,1,2$.

\section{References}

[1] Y. Achdou, C. Bernardi, and F. Coquel. A priori and a posteriori analysis of finite volume discretizations of Darcy's equations. Numer. Math., 96(1):17-42, 2003.

[2] M. Ainsworth. A posteriori error estimation for lowest order Raviart-Thomas mixed finite elements. SIAM J. Sci. Comput., 30(1):189-204, 2007.

[3] T. Arbogast and Z. Chen. On the implementation of mixed methods as nonconforming methods for second-order elliptic problems. Math. Comp., 64(211):943-972, 1995.

[4] M. Arioli. A stopping criterion for the conjugate gradient algorithms in a finite element method framework. Numer. Math., 97(1):1-24, 2004.

[5] M. Arioli and D. Loghin. Stopping criteria for mixed finite element problems. Electron. Trans. Numer. Anal., 29:178-192, 2007/08.

[6] D. N. Arnold and F. Brezzi. Mixed and nonconforming finite element methods: implementation, postprocessing and error estimates. RAIRO Modél. Math. Anal. Numér., 19(1):7-32, 1985.

[7] R. Becker, C. Johnson, and R. Rannacher. Adaptive error control for multigrid finite element methods. Computing, 55(4):271-288, 1995. 

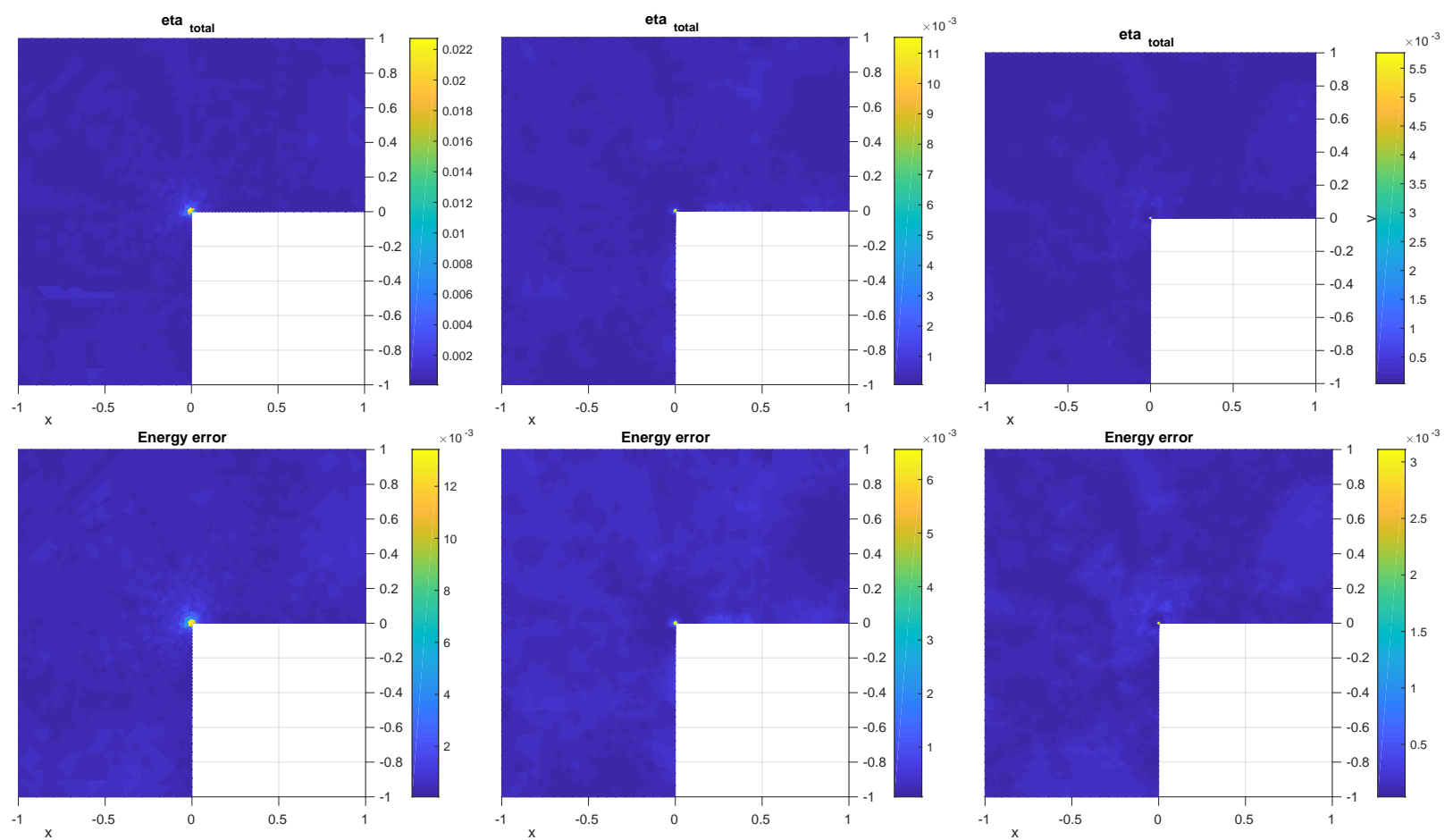

Figure 16: Example 3: the distribution of the total error estimator (top) and of the energy error (bottom) for different refinements: $\ell=0$ (left), $\ell=1$ (middle), and $\ell=2$ (right).

[8] E. Burman and A. Ern. Continuous interior penalty $h p$-finite element methods for advection and advection-diffusion equations. Math. Comp., 76(259):1119-1140, 2007.

[9] S.-L. Campbell, I.-C.-F. Ipsen, C.-T. Kelley, and C.-D. Meyer. GMRES and the minimal polynomial. BIT, 4(36):664-675, 1996.

[10] C. Cancès, I. S. Pop, and M. Vohralík. An a posteriori error estimate for vertex-centered finite volume discretizations of immiscible incompressible two-phase flow. Math. Comp., 83(285):153-188, 2014.

[11] P. Ciarlet, Jr., E. Jamelot, and F. D. Kpadonou. Domain decomposition methods for the diffusion equation with low-regularity solution. Comput. Math. Appl., 74(10):2369-2384, 2017.

[12] L. C. Cowsar, J. Mandel, and M. F. Wheeler. Balancing domain decomposition for mixed finite elements. Math. Comp., 64(211):989-1015, 1995.

[13] F. Cuvelier. Personal communication.

[14] D. A. Di Pietro, E. Flauraud, M. Vohralík, and S. Yousef. A posteriori error estimates, stopping criteria, and adaptivity for multiphase compositional Darcy flows in porous media. J. Comput. Phys., 276:163-187, 2014.

[15] V. Dolean, P. Jolivet, and F. Nataf. An introduction to domain decomposition methods. Society for Industrial and Applied Mathematics (SIAM), Philadelphia, PA, 2015.

[16] V. Dolejší, A. Ern, and M. Vohralík. $h p$-adaptation driven by polynomial-degree-robust a posteriori error estimates for elliptic problems. SIAM J. Sci. Comput., 38(5):A3220-A3246, 2016.

[17] J. Douglas, Jr., P. J. Paes-Leme, J. E. Roberts, and J. P. Wang. A parallel iterative procedure applicable to the approximate solution of second order partial differential equations by mixed finite element methods. Numer. Math., 65(1):95-108, 1993.

[18] A. Ern and M. Vohralík. Adaptive inexact Newton methods with a posteriori stopping criteria for nonlinear diffusion PDEs. SIAM J. Sci. Comput., 35(4):A1761-A1791, 2013. 
[19] A. Ern and M. Vohralík. Polynomial-degree-robust a posteriori estimates in a unified setting for conforming, nonconforming, discontinuous Galerkin, and mixed discretizations. SIAM J. Numer. Anal., 53(2):1058-1081, 2015.

[20] C. Farhat and F.-X. Roux. A method of finite element tearing and interconnecting and its parallel solution algorithm. Internat. J. Numer. Methods Engrg., 32(6):1205-1227, 1991.

[21] M. J. Gander. Optimized Schwarz methods. SIAM J. Numer. Anal., 44(2):699-731, 2006.

[22] M. J. Gander and O. Dubois. Optimized Schwarz methods for a diffusion problem with discontinuous coefficient. Numer. Algorithms, 69(1):109-144, 2015.

[23] M.-G. Gasparo, A. Papini, and A. Pasquali. Some properties of GMRES in Hilbert spaces. Numerical Functional Analysis and Optimization, 29(11-12):276-1285, 2014.

[24] F. Hecht. FreeFem ++ . Technical report, Lab. Jacques-Louis Lions, Univ. Pierre et Marie Curie, Paris, http://www.freefem.org/ff++/ftp/freefem++doc.pdf, 2017.

[25] T.-T.-P. Hoang, J. Jaffré, C. Japhet, M. Kern, and J. E. Roberts. Space-time domain decomposition methods for diffusion problems in mixed formulations. SIAM J. Numer. Anal., 51(6):3532-3559, 2013.

[26] T. T. P. Hoang, C. Japhet, M. Kern, and J. Roberts. Ventcell conditions with mixed formulations for flow in porous media. In Domain decomposition methods in science and engineering XXII, volume 104 of Lect. Notes Comput. Sci. Eng., pages 531-540. Springer, Cham, 2016.

[27] C. Japhet and F. Nataf. The best interface conditions for domain decomposition methods: absorbing boundary conditions. In Absorbing Boundaries and Layers, Domain Decomposition Methods, pages 348-373. Nova Sci. Publ., Huntington, NY, 2001.

[28] C. Japhet, F. Nataf, and F. Rogier. The optimized order 2 method. Application to convection-diffusion problems. Future Gener. Comp. Sy., 18(1):17-30, 2001.

[29] P. Jiránek, Z. Strakoš, and M. Vohralík. A posteriori error estimates including algebraic error and stopping criteria for iterative solvers. SIAM J. Sci. Comput., 32(3):1567-1590, 2010.

[30] O. A. Karakashian and F. Pascal. A posteriori error estimates for a discontinuous Galerkin approximation of second-order elliptic problems. SIAM J. Numer. Anal., 41(6):2374-2399, 2003.

[31] K. Y. Kim. A posteriori error analysis for locally conservative mixed methods. Math. Comp., 76(257):43$66,2007$.

[32] P. Ladevèze and J.-P. Pelle. Mastering calculations in linear and nonlinear mechanics. Mechanical Engineering Series. Springer-Verlag, New York, 2005. Translated from the 2001 French original by Theofanis Strouboulis.

[33] J.-L. Lions and E. Magenes. Problèmes aux limites non homogènes et applications. Vol. 1. Travaux et Recherches Mathématiques, No. 17. Dunod, Paris, 1968.

[34] P.-L. Lions. On the Schwarz alternating method. III: a variant for nonoverlapping subdomains. In T. et al., editor, Third International Symposium on Domain Decomposition Methods for Partial Differential Equations (Houston, TX, 1989), pages 202-223. Philadelphia, PA, SIAM, 1990.

[35] J. Mandel. Balancing domain decomposition. Comm. Numer. Methods Engrg., 9(3):233-241, 1993.

[36] J. Mandel and M. Brezina. Balancing domain decomposition for problems with large jumps in coefficients. Math. Comp., 65(216):1387-1401, 1996.

[37] D. Meidner, R. Rannacher, and J. Vihharev. Goal-oriented error control of the iterative solution of finite element equations. J. Numer. Math., 17(2):143-172, 2009.

[38] A. T. Patera and E. M. Rønquist. A general output bound result: application to discretization and iteration error estimation and control. Math. Models Methods Appl. Sci., 11(4):685-712, 2001. 
[39] G. V. Pencheva, M. Vohralík, M. F. Wheeler, and T. Wildey. Robust a posteriori error control and adaptivity for multiscale, multinumerics, and mortar coupling. SIAM J. Numer. Anal., 51(1):526-554, 2013.

[40] W. Prager and J. L. Synge. Approximations in elasticity based on the concept of function space. Quart. Appl. Math., 5:241-269, 1947.

[41] A. Quarteroni and A. Valli. Domain decomposition methods for partial differential equations. Numerical Mathematics and Scientific Computation. Oxford University Press, New York, 1999. Oxford Science Publications.

[42] S. Repin. A posteriori estimates for partial differential equations, volume 4 of Radon Series on Computational and Applied Mathematics. Walter de Gruyter GmbH \& Co. KG, Berlin, 2008.

[43] V. Rey, P. Gosselet, and C. Rey. Strict lower bounds with separation of sources of error in nonoverlapping domain decomposition methods. Internat. J. Numer. Methods Engrg., 108(9):1007-1029, 2016.

[44] V. Rey, C. Rey, and P. Gosselet. A strict error bound with separated contributions of the discretization and of the iterative solver in non-overlapping domain decomposition methods. Comput. Methods Appl. Mech. Engrg., 270:293-303, 2014.

[45] Y. Saad. Iterative methods for sparse linear systems. Society for Industrial and Applied Mathematics, Philadelphia, PA, second edition, 2003.

[46] Y. Saad and M. H. Schultz. GMRES: a generalized minimal residual algorithm for solving nonsymmetric linear systems. SIAM J. Sci. Statist. Comput., 7(3):856-869, 1986.

[47] H. A. Schwarz. Gesammelte mathematische Abhandlungen. Band I, II. Chelsea Publishing Co., Bronx, N.Y., 1972. Nachdruck in einem Band der Auflage von 1890.

[48] M. Vohralík. A posteriori error estimates for lowest-order mixed finite element discretizations of convection-diffusion-reaction equations. SIAM J. Numer. Anal., 45(4):1570-1599, 2007.

[49] M. Vohralík. Unified primal formulation-based a priori and a posteriori error analysis of mixed finite element methods. Math. Comp., 79(272):2001-2032, 2010. 